\title{
Fully-resolved simulations of particle-laden viscoelastic fluids using an immersed boundary method
}

\author{
C. Fernandes ${ }^{a, *}$, S.A. Faroughi ${ }^{b}$, O.S. Carneiro ${ }^{a}$, J. Miguel Nóbrega ${ }^{a}$, G.H. McKinley ${ }^{b}$ \\ a Institute for Polymers and Composites/i3N, University of Minho, Campus de Azurém, 4800-058 Guimarães, Portugal \\ ${ }^{\mathrm{b}}$ Hatsopoulos Microfluids Laboratory, Department of Mechanical Engineering, Massachusetts Institute of Technology, Cambridge, MA, 02139, US
}

\section{A R T I C L E I N F O}

\section{Keywords:}

Particle-laden flow

Viscoelastic fluid

Finite volume method

Immersed boundary method

Fully resolved simulations

\begin{abstract}
A B S T R A C T
This study reports the development of a direct simulation code for solid spheres moving through viscoelastic fluids with a range of different rheological behaviors. The numerical algorithm was implemented on an opensource finite-volume solver coupled with an immersed boundary method, and is able to perform fully-resolved simulations, wherein all flow scales associated with the particle motion are resolved. The formulation employed exploits the log-conformation tensor to avoid high Weissenberg number issues when calculating the polymeric extra stress. A number of benchmark flows were simulated using this method, to assess the accuracy of the newlydeveloped solver. First, the sedimentation of a sphere in a bounded domain surrounded by either Newtonian or viscoelastic fluid was computed, and the numerical results were verified by comparison with experimental and computational data from the literature. Additionally, the spatial and temporal accuracies of the algorithm were evaluated, and different transient and advection discretization schemes were investigated. Second, the rotation of a sphere in a homogeneous shear flow was studied, and again the numerical results obtained were compared to those from the literature. Good agreement is obtained for the variation in the particle rotation rate as a function of Weissenberg number, using both the newly implemented algorithm and an alternative fixed-mesh approach. Finally, the cross-stream migration of a neutrally buoyant sphere in a steady Poiseuille flow, consisting of either a Newtonian or viscoelastic suspending fluid was investigated. For the Newtonian fluid good agreement was obtained for the particle equilibrium position when compared to the well known Segré-Silberberg effect, and for the viscoelastic fluid the effect of the retardation ratio on the final particle equilibrium position was studied. Additionally, the newly-developed solver capabilities were tested to study the shear-induced particle alignment in wall-bounded Newtonian and viscoelastic fluids. The role of the fluid rheology and finite gap size on both the rate and approach pathways of the solid particles is illustrated.
\end{abstract}

\section{Introduction}

Fluid-particle transport problems occur in many different forms, and with significant practical relevance, in several engineering applications, such as oil sands mining, fluidized beds, coal-based combustion chambers and biomass gasifiers [1-4]. In many applications it is essential to consider that the fluid, in which the particles are dispersed, has underlying viscoelastic characteristics.

The use of numerical simulations to understand the behavior of multiphase flows, including those with viscoelastic matrix fluids, provides a very important source of insight into the physical transport processes that occur between freely-moving particles and nonlinear fluids. Amongst others, one commonly method employed to solve such simulations is the Computational Fluid Dynamics - Discrete Element Method (CFD-DEM) [5], which in turn can be categorized as following a resolved or unresolved approach, depending on the size of the particles relative to the smallest computational mesh cell size. In resolved CFDDEM $[6,7]$ the particles are substantially larger than the individual computational cells, i.e., when represented within the mesh a particle covers multiple cells. Due to limitations on computational capabilities this method can only be used for cases where relatively small number of particles, say a few hundreds or $\mathrm{O}(1000)$ particles, need to be considered. The fluid field around each particle is resolved with a detailed mesh, and the force balance on each particle is calculated individually, which provides a comprehensive understanding of the underlying physics [812]. This approach belongs to the class of Direct Numerical Simulations (DNS) $[13,14]$. In contrast, unresolved CFD-DEM [15] is designed for handling very large numbers of particles, which should be significantly smaller than the computational mesh cells. Consequently, each cell can contain several particles. For such simulations it is essential to know

\footnotetext{
* Corresponding author.

E-mail addresses: cbpf@dep.uminho.pt (C. Fernandes), faroughi@mit.edu (S.A. Faroughi), olgasc@dep.uminho.pt (O.S. Carneiro), mnobrega@dep.uminho.pt (J.M. Nóbrega), gareth@mit.edu (G.H. McKinley).
} 
how the stress in a single cell depends on both the volume fraction of particles and the type of suspending fluid.

With increasing computational power the DNS (resolved CFD-DEM) of fluid-particle problems has steadily gained importance. The first person to use such a method was Peskin $[13,14]$, who studied the flow patterns around heart valves by using the so-called Immersed Boundary (IB) method. The generic term IB or Fictitious Domain Method (FDM), summarizes all kinds of methods that are suitable for describing complex structures within the flow domain of interest, and the no-slip boundary condition at the solid-particle surface appears as a source-term in the momentum equations. The key idea behind these methods is to use a fixed Eulerian mesh for the computation and to represent the immersed object using a Lagrangian mesh, which is free to move inside the Eulerian mesh. This fixed-grid approach makes it possible to efficiently simulate many-particle systems, from the computational point of view. Glowinski et al. [16-18] were the first to introduce a FDM that made use of Distributed Lagrange Multipliers (DLM). In their concept, they incorporated Lagrangian multipliers as a constraint to the weak form of the Navier-Stokes equations, enforcing the boundary conditions of the rigid body in the system. In their original work, the Lagrangian multiplier was calculated explicitly, which made the method rather inefficient [19]. Patankar et al. [6], Diaz-Goano et al. [20] and Yu and Shao [21] improved the method by using body forces, which enforced the rigidity constraint, and therefore avoided the necessity of explicitly calculating the Lagrangian multiplier. These methods have been tested in simulations with Newtonian fluids (with volume fractions in the range $0.36 \leq \phi \leq 0.61$ ) and good agreement with experimental data was found $[22,23]$.

The literature regarding IB studies with viscoelastic fluids is limited and began with the work of Bodart and Crochet [24], who used the Finite Element Method (FEM) to simulate the motion of a rigid spherical particle sedimenting in a circular cylinder filled with an Oldroyd-B fluid. Later, Binous and Phillips [25] performed simulations of a single sphere, a single non-spherical particle, and two spheres sedimenting in viscoelastic suspensions of FENE dumbbells, using a modified version of the Stokesian dynamics method. Singh et al. [26] used a DLM method for simulating the motion of rigid cylindrical particles suspended in an Oldroyd-B fluid in a channel geometry, to study the alignment of the particles close to the walls. Ardekani et al. [27] presented experimental results on particle-wall collisions in viscoelastic fluids, and later, Li et al. [28] studied numerically the migration of a sphere in the pressuredriven channel flow of viscoelastic fluids modeled by the Oldroyd-B and Giesekus equations of state. The effects of inertia, elasticity, shearthinning viscosity, secondary flows and the blockage ratio were considered. Additionally, Li et al. [29] and Li and Ardekani [30] studied numerically the effects of non-Newtonian fluid properties and solid boundaries on the swimming dynamics of microorganisms. Recently, Villone et al. [31] studied the cross-streamline migration of a spherical particle flowing in a wide micro-slit device containing a viscoelastic fluid, which was modeled by either the Giesekus or the Phan Thien-Tanner constitutive equations, through three-dimensional FEM simulations. Goyal and Derksen [32] described coupled lattice-Boltzmann and IB methods for studying particles sedimenting in a FENE-CR fluid. Finally, Krishnan et al. [12] used an unstructured mesh code with an IB based viscoelastic solver for moving bodies in a FVM numerical algorithm. Common to all the methods presented above is the fact that they are all developed as in-house codes, which limits their easy applicability to other scientific areas, and are typically limited to low Weissenberg number problems $(W i \leq 5)$.

To the best of the authors' knowledge, the combined use of a threedimensional unstructured mesh code, with a log-conformation approach to enable access to high Weissenberg number flows, and an IB method for rigid bodies moving in viscoelastic fluids has not been presented to date. The present work aims to develop and validate a numerical code that is able to simulate the flow behavior of particle-laden viscoelastic fluids, by extending the open-source computational fluid dynamics library CFDEMcoupling [33] to include calculations with viscoelastic fluids and with the log-conformation approach. The use of a common opensource computational library to perform these new developments is expected to increase the accessibility and utility of the contribution. The numerical method implemented is based on the approaches described in Shirgaonkar et al. [7], Hager et al. [34] and Aycock et al. [35], where the latter study presents an improved IB method to describe the flow of Newtonian fluids around spherical particles. To verify the robustness of the implemented numerical approach, a number of benchmark problems with known published results were simulated. Additionally, the accuracy of the algorithm was evaluated, both in terms of spatial and temporal resolutions, by performing extrapolation of the results to the limit of an infinitely refined mesh. The fluids used in the present work are modeled by different constitutive rheological models, namely Chilcott and Rallison's Finitely Extensible Non-linearly Elastic dumbbell (FENE-CR) model [36], the quasi-linear elastic dumbbell model (Oldroyd-B) [37] and the configuration-dependent molecular mobility model (Giesekus) [38]. A wide range of fluid elasticities, including high Weissenberg number flows, can be attained due to incorporation of the log-conformation approach $[39,40]$ for calculating the polymeric extrastress tensor.

This paper describes the numerical implementation of fully-resolved simulations, i.e. a DNS based approach. To accomplish the required developments an open-source library, CFDEMcoupling [33], was modified to be able to calculate flow of viscoelastic fluids and the resulting hydrodynamic loads on the particles, that, in turn, determine their linear and rotational motions. The latter information is fed back to the fluid flow as moving no-slip boundary conditions that are imposed on the particles surface.

The paper is organized in the following manner: in Section 2 we present the mathematical formulation of the viscoelastic IB code that is developed in this work, including the governing equations and coupling of both continuum and discrete phases. Section 3 provides the details of the numerical discretization and solution procedure for the overall algorithm that is described by the mathematical formulation presented in Section 2. In Section 4 we present a number of case studies involving Newtonian and viscoelastic flows around spherical particles, namely the sedimentation of a sphere in a bounded domain [41,42], the rotation of a sphere suspended in a homogeneous steady shear flow of an Oldroyd-B fluid [43], and the cross-stream migration of a neutrallybuoyant sphere in Poiseuille flow $[35,44]$. In each case, a comparison of the results obtained with the newly-developed code is performed with data reported in the literature, in order to assess both accuracy and robustness. Section 5 is dedicated to illustrating the capability of the code to solve a physical challenging problem, the shear-induced particle alignment in wall-bounded Newtonian and viscoelastic fluids. Finally, in Section 6, we summarize the main conclusions of this work.

\section{Mathematical formulation}

In this section we present the mathematical formulation for an algorithm that is able to efficiently handle the rigid body motion of particulate spherical bodies surrounded by a viscoelastic fluid. The algorithm considers a constraint-based formulation, which provides a rigorous basis for the IB implementation performed in the open-source framework code CFDEMcoupling [34,35].

The open-source IB solver originally developed by Hager et al. [34], and later modified by Aycock et al. [35] to incorporate two-way rotation coupling (here denoted CFD/6-DOF), was modified and improved for the present study to incorporate suspending fluids governed by viscoelastic constitutive equations.

\subsection{Governing equations}

Consider the total computational domain $\Omega\left(=\Omega_{s} \cup \Omega_{f}\right)$, including the solid ("body") domain $\Omega_{s}$ and the fluid domain $\Omega_{f}$, whose 
boundaries are represented by $\partial \Omega, \partial \Omega_{s}$ and $\partial \Omega_{f}$, respectively. The equations of motion for the fluid domain are governed by the conservation of mass,

$\nabla \cdot \mathbf{u}=0$ in $\Omega_{f}$

conservation of momentum,

$\rho_{f}\left(\frac{\partial \mathbf{u}}{\partial t}+\mathbf{u} \cdot \nabla \mathbf{u}\right)-\left(\eta_{S}+\eta^{\star}\right) \nabla^{2} \mathbf{u}=-\nabla p-\eta^{\star} \nabla \cdot \nabla \mathbf{u}+\nabla \cdot \tau_{P}$ in $\Omega_{f}$

plus an appropriate constitutive equation for the polymeric extra-stress tensor $\tau_{P}$. Here $\rho_{f}$ and $\mathbf{u}$ are the fluid density and velocity vector, respectively, $t$ is the time, $p$ is the pressure, $\eta_{S}$ is the viscosity of the Newtonian suspending solvent and $\eta^{\star}$ is an artificial viscosity proportional to the polymeric viscosity, $\eta_{P}$, coming from the employment of a stabilizing approach, known as improved both-sides diffusion method (iBSD) [45]. In the present work three different constitutive models were considered, namely, the Oldroyd-B model [37],

$\lambda \stackrel{\nabla}{\tau}_{P}+\tau_{P}=\eta_{P}\left(\nabla \mathbf{u}+\nabla \mathbf{u}^{T}\right)$

the FENE-CR model [36],

$\frac{\lambda}{\frac{L^{2}+\left(\lambda / \eta_{P}\right) \operatorname{tr}\left(\tau_{P}\right)}{L^{2}-3}} \stackrel{\nabla}{\tau}_{P}+\boldsymbol{\tau}_{P}=\eta_{P}\left(\nabla \mathbf{u}+\nabla \mathbf{u}^{T}\right)$

and the Giesekus model [38],

$\lambda \stackrel{\nabla}{\tau_{P}}+\tau_{P}+\frac{\alpha \lambda}{\eta_{P}} \tau_{P} \cdot \tau_{P}=\eta_{P}\left(\nabla \mathbf{u}+\nabla \mathbf{u}^{T}\right)$

In the constitutive model equations, $\lambda$ is the fluid relaxation time, $L^{2}$ is the extensibility parameter $(L \rightarrow \infty$ reduces Eq. (4) to the Oldroyd-B model Eq. (3)), $\alpha$ is the mobility parameter, $\operatorname{tr}(\cdot)$ is the trace operator and $\stackrel{\nabla}{\tau}_{P}$ indicates the upper-convected time derivative of the polymeric extra-stress tensor defined as

$\stackrel{\nabla}{\tau}_{P} \equiv \frac{\partial \tau_{P}}{\partial t}+\mathbf{u} \cdot \nabla \boldsymbol{\tau}_{P}-\tau_{P} \cdot \nabla \mathbf{u}-\nabla \mathbf{u}^{T} \cdot \boldsymbol{\tau}_{P}$

For closure purposes, the system of governing equations requires appropriate boundary conditions:

$\mathbf{u}=\mathbf{u}_{\partial \Omega} \quad$ on $\quad \partial \Omega$

the interface conditions on the fluid-solid boundary,

$\mathbf{u}=\mathbf{u}_{i} \quad$ and $\quad\left(-p \mathbf{I}+\eta_{S}\left(\nabla \mathbf{u}+\nabla \mathbf{u}^{T}\right)+\tau_{P}\right) \cdot \hat{\mathbf{n}}=\mathbf{t}_{\partial \Omega_{s}} \quad$ on $\quad \partial \Omega_{s}$

and the initial condition,

$\mathbf{u}(\mathbf{x}, t=0)=\mathbf{u}_{0}(\mathbf{x}) \quad$ in $\quad \Omega_{f}$

In the above equations $\hat{\mathbf{n}}$ is the outward normal unit vector to $\partial \Omega_{s}, \mathbf{t}_{\partial \Omega_{s}}$ is the traction vector acting from the fluid on the solid body surface and $\mathbf{u}_{i}$ is the (unknown) velocity of the solid-fluid interface. The initial velocity $\mathbf{u}_{0}$ is required to satisfy Eq. (1) and the boundary velocity in Eq. (7) should satisfy the compatibility condition due to Eq. (1) at all times.

When written in the continuum formulation, viscoelastic fluids in high Weissenberg number flows are well known to introduce numerical convergence difficulties in computational codes, which are mainly related to the lack of sufficient resolution of discretization methods to resolve the exponential growth of stresses near critical points as the Weissenberg number is incremented [39]. In the present work, for the calculation of the polymeric extra-stress tensor components, we follow the implementation of the log-conformation approach in the OpenFOAM [46] computational library, presented in Habla et al. [47] and Pimenta and Alves $[48,49]$. Details on the mathematical formulation behind the log-conformation approach can be found in the original works of Fattal and Kupferman $[39,40]$.

For the dispersed solid phase, the DEM, firstly presented by Cundall and Strack [50], is used to solve the equations for conservation of linear and angular momenta, governing the motion of a particle $i$ of mass $m_{i}$ :

$$
\begin{aligned}
m_{i} \frac{d \mathbf{U}_{i}^{p}}{d t} & =\sum_{j=1}^{n_{i}^{c}} \mathbf{F}_{i j}^{c}+\mathbf{F}_{i}^{f}+\mathbf{F}_{i}^{g} \\
I_{i} \frac{d \boldsymbol{\omega}_{i}}{d t} & =\sum_{j=1}^{n_{i}^{c}} \mathbf{M}_{i j}^{c}+\mathbf{M}_{i}^{f}
\end{aligned}
$$

where $\mathbf{U}_{i}^{p}$ and $\omega_{i}$ denote the translational and angular velocities of particle $i$, respectively, $\mathbf{F}_{i j}^{c}$ and $\mathbf{M}_{i j}^{c}$ are, respectively, the contact force and contact torque that result from particle interaction or from the contact with walls, with $n_{i}^{c}$ the number of total contacts for particle $i, \mathbf{F}_{i}^{f}$ and $\mathbf{M}_{i}^{f}$ are the particle-fluid interaction force and the torque acting on particle $i$, respectively, $\mathbf{F}_{i}^{g}$ is the buoyancy force, and $I_{i}$ is the moment of inertia of particle $i$.

\subsection{CFD and DEM coupling}

The fluid governing equations, Eqs. (1)-(9), are coupled and must be solved in conjunction with the equations for the motion of the solid particle(s), Eqs. (10) and (11). The equations of motion for the fluid and the particles are coupled through the interface conditions given by Eq. (8). The first equality in Eq. (8) is used to transfer the particle velocity to the fluid velocity field. The second describes the stress or traction at the interface between the fluid and the solid and can be transformed to a drag force term as follows. First, we integrate the stress interface condition over the particle boundary,

$\int_{\partial \Omega_{s}}\left(-p \mathbf{I}+\eta_{S}\left(\nabla \mathbf{u}+\nabla \mathbf{u}^{T}\right)+\tau_{P}\right) \cdot \hat{\mathbf{n}} d \partial \Omega_{s}=\int_{\partial \Omega_{s}} \mathbf{t}_{\partial \Omega_{s}} d \partial \Omega_{s}$

By applying the Gauss divergence theorem we obtain,

$\int_{\Omega_{s}} \nabla \cdot\left(-p \mathbf{I}+\eta_{S}\left(\nabla \mathbf{u}+\nabla \mathbf{u}^{T}\right)+\tau_{P}\right) d \Omega_{s}=\int_{\partial \Omega_{s}} \mathbf{t}_{\partial \Omega_{s}} d \partial \Omega_{s}$

which can be rewritten as,

$\int_{\Omega_{s}}\left[-\nabla p+\nabla \cdot\left(\eta_{S}\left(\nabla \mathbf{u}+\nabla \mathbf{u}^{T}\right)\right)+\nabla \cdot \tau_{P}\right] d \Omega_{s}=\int_{\partial \Omega_{s}} \mathbf{t}_{\partial \Omega_{s}} d \partial \Omega_{s}$

Making use of the identity $\nabla \cdot\left(\nabla \mathbf{u}+(\nabla \mathbf{u})^{T}\right)=\nabla^{2} \mathbf{u}+\nabla(\nabla \cdot \mathbf{u})$ and knowing that, due to mass conservation, $\nabla \cdot \mathbf{u}=0$, we can rewrite Eq. (14) as,

$\int_{\partial \Omega_{s}} \mathbf{t}_{\partial \Omega_{s}} d \partial \Omega_{s}=\int_{\Omega_{s}}\left[-\nabla p+\eta_{S} \nabla^{2} \mathbf{u}+\nabla \cdot \tau_{P}\right] d \Omega_{s}$

Eq. (15) shows that the fluid forces acting on the particles consist of contributions from the pressure, from viscous stress as well as from the polymeric contribution to the stress field.

\subsection{Force and torque calculation}

This section describes the procedure used to compute both the forces and torques, which are present on the right hand side of Eqs. (10) and (11). For that, Eq. (15) needs to be discretized in order to enable computation of the drag force $\mathbf{F}_{i}^{f}$. Let $\mathbf{x}$ be an arbitrary region from $\Omega$. Then,

$\int_{\Omega_{s}}-\nabla p+\eta_{S} \nabla^{2} \mathbf{u}+\nabla \cdot \tau_{P} d \Omega_{s}=\int_{\Omega}\left(-\nabla p+\eta_{S} \nabla^{2} \mathbf{u}+\nabla \cdot \tau_{P}\right) \delta_{\Omega} d \Omega$

where $\delta_{\Omega}=1$ if $\mathbf{x} \in \Omega_{s}$, and $\delta_{\Omega}=0$ otherwise. Assuming that $T_{h}$ is a decomposition of $\Omega$ consisting of computational cells $c$, we can write

$$
\begin{aligned}
& \int_{\Omega}\left(-\nabla p+\eta_{S} \nabla^{2} \mathbf{u}+\nabla \cdot \boldsymbol{\tau}_{P}\right) \delta_{\Omega} d \Omega \\
& =\sum_{c \in T_{h}} \int_{V(c)}\left(-\nabla p+\eta_{S} \nabla^{2} \mathbf{u}+\nabla \cdot \tau_{P}\right) \delta_{\Omega} d V(c)
\end{aligned}
$$

where $V(c)$ is the volume of cell $c$. Notice that for notation purposes we use the parentheses $(c)$ to evaluate a function on cell $c$. Numerical 
integration of Eq. (17) leads to the final form of the fluid force acting on the particle,

$\mathbf{F}_{i}^{f}=\sum_{c \in \bar{T}_{h}}\left(-\nabla p+\eta_{S} \nabla^{2} \mathbf{u}+\nabla \cdot \boldsymbol{\tau}_{P}\right)(c) \cdot V(c)$

where $\bar{T}_{h}$ is the set of all cells covered, in full or in part, by a particle.

Following a similar procedure, the fluid torques $\left(\mathbf{M}_{i}^{f}\right)$ can be calculated by taking the cross product between the position vector $\mathbf{r}$ (pointing from the fluid cell centroid to the particle centroid) and the total stress from Eq. (18). This leads to

$\mathbf{M}_{i}^{f}=\sum_{c \in \bar{T}_{h}}\left[\mathbf{r}(c) \times\left(-\nabla p+\eta_{S} \nabla^{2} \mathbf{u}+\nabla \cdot \boldsymbol{\tau}_{P}\right)(c)\right] \cdot V(c)$

Notice that the load contribution arising from pressure does not give rise to any torque contribution, because we are dealing here exclusively with spheres. Thus, normal loads acting perpendicular to the particle surface, such as pressure, do not induce any torque.

The buoyancy force $\left(\mathbf{F}_{i}^{g}\right)$ is given by the weight of the displaced fluid and can be calculated by numerically integrating the fluid density $\left(\rho_{f}\right)$ over the volume of the solid region in the mesh $\left(V(c)\right.$ with $\left.c \in \bar{T}_{h}\right)$, to obtain the total displaced fluid mass $\left(\rho_{f} V(c)\right.$ with $\left.c \in \bar{T}_{h}\right)$. Next, multiplying the fluid mass by the gravitational acceleration vector $(\mathbf{g})$, we obtain the buoyancy force

$\mathbf{F}_{i}^{g}=\sum_{c \in \bar{T}_{h}}\left(\rho_{f} \mathbf{g}\right)(c) \cdot V(c)$

The contact force $\left(\mathbf{F}_{i j}^{c}\right)$ and torque $\left(\mathbf{M}_{i j}^{c}\right)$ are calculated using the nonlinear elastic Hertz-Mindlin contact model [5,51]. Details about this model and its implementation within the DEM numerical algorithm can be found elsewhere [5,52].

\section{Calculation procedure}

Numerical solution of the equations presented in Section 2 relies on a collocated FVM approach, which uses the conservative integral forms of the governing equations for the fluid phase [53]. In this method, the spatial derivatives over the volume are converted into integrals over surfaces in terms of the flux, the time derivative is semi-discretized (also called the method of lines [54]), the grid points define the faces, the data is stored at the center of the computational cells' control volume, the fluxes are obtained by velocities interpolated to the faces at each time step, and the integrals are evaluated by the use of the mean value theorem. The implementation of the different conservation equations involved (continuity, momentum and constitutive equations) is done using the CFDEMcoupling framework [33], based on the OpenFOAM computational library $[46,55]$. The solution procedure adopted to compute the viscoelastic IB equations presented in Section 2 is described next.

The algorithm (see Fig. 1) is implemented assuming a body-fitted mesh, for which the OpenFOAM [46] dynamic mesh capabilities (dynamicRefineFvMesh) [56] are used to refine the mesh near the solid-fluid interface. In this work we studied the influence of the dynamic mesh maxRefinement parameter (here denoted dyM_max), which defines the maximum number of layers of refinement that a cell can experience, on the accuracy of the results obtained. Moreover, the algorithm uses a mixed Eulerian-Lagrangian formulation in which the solid object is tracked by a collection of Lagrangian particles and the fluid is discretized on a Eulerian grid. The algorithm follows three main steps: a) the fluid equations are solved on the whole computational domain, b) the rigid motion of the solid bodies is computed with LIGGGHTS [57] and incorporated in the form of a body force and, finally, c) we apply the divergence-free condition on the velocity field obtained on the last step, and correct the pressure and velocity fields. The presented algorithm follows the ideas of Patankar et al. [6] and Shirgaonkar et al. [7], with extension to viscoelastic fluid flows where the polymeric extra-stress tensor components are calculated based on the log-conformation approach $[39,40]$.
The numerical calculation procedures are carried out as follows:

1. At $t=0$ the fluid and particle initial and boundary conditions are read from the case study input files. Additionally, the DEM solver sends the particle initial position and velocities to the CFD solver.

2. The time iteration starts, $0<t<$ endTime.

3. The particles are located within the Eulerian mesh. The cell ID of the centre position of each particle is saved. Additionally, the particle volume fraction in each cell is computed.

4. If chosen, the dynamic mesh capabilities (dynamicRefineFvMesh) are used to refine the Eulerian mesh at the particle-fluid interface region.

5. Using the fluid solution from the last time-step in the regions marked by the particle volume fraction, the fluid loads, which act on each particle, are computed $\left(\mathbf{F}_{i}^{f}, \mathbf{F}_{i}^{g}, \mathbf{M}_{i}^{f}\right)$. The resulting loads for each particle are returned to the DEM solver.

6. A data exchange model is used to run a DEM script, which will compute the particles positions and velocities (Eqs. (10) and (11)), using Velocity-Verlet integration [58]. Additionally, if collisions between particles or particle-wall are detected, the collision loads are computed $\left(\mathbf{F}_{i j}^{c}, \mathbf{M}_{i j}^{c}\right)$. Notice that the code accounts for an independent choice of the time-step size in CFD and DEM, and the only thing which needs to be guaranteed is that the time steps and coupling steps match. For all the simulations presented hereafter the DEM time-step size is $1 / 10$ th of the CFD time-step size.

7. The new particle position and the translational and angular velocities are transferred to the CFD solver.

8. The CFD solver proceeds with the PISO (Pressure-Implicit with Splitting of Operators) algorithm [59], which solves the fluid constitutive equations (Eqs. (1)-(6)) by using the log-conformation approach $[39,40]$. An intermediate velocity field $\hat{\mathbf{u}}$ is obtained by solving the momentum balance equations (Eq. (2)) and an intermediate pressure $\tilde{p}$ is obtained from the continuity equation (Eq. (1)), which gives a Poisson equation for pressure correction.

9. The next step is to correct the intermediate velocity field $\widehat{\mathbf{u}}$ in the region marked by the particle volume fraction, imposing the rigid component of the body velocity provided by the DEM calculation. This correction is equivalent to adding a body force per unit volume term $\mathbf{f}$ in the semi-discrete Cauchy momentum equations, to obtain a corrected velocity field $\widetilde{\mathbf{u}}$ :

$\mathbf{f}=\rho \frac{\partial}{\partial t}(\tilde{\mathbf{u}}-\widehat{\mathbf{u}})$

with $\widetilde{\mathbf{u}}=\mathbf{U}_{i}^{p}+\boldsymbol{\omega}_{i} \times \mathbf{r}$ defined only for the cells within the solid body. The translational and angular velocities, $\mathbf{U}_{i}^{p}$ and $\omega_{i}$, respectively, were previously computed in step 6 .

10. The previous step introduces a discontinuity in velocity at the interface, giving rise to a non-zero divergence at the fluid-solid interface. Thus $\widetilde{\mathbf{u}}$ is projected onto a divergence-free velocity space $\overline{\mathbf{u}}$, by using a scalar field $\phi$, as:

$\overline{\mathbf{u}}=\widetilde{\mathbf{u}}-\nabla \phi$

where $\phi$ is obtained by solving the Poisson equation,

$\nabla^{2} \phi=\nabla \cdot \widetilde{\mathbf{u}}$

11. Then $\overline{\mathbf{u}}$ is calculated by Eq. (22).

12. The last step is equivalent to adding a pressure force $-\rho \frac{\nabla \phi}{\Delta t}$ in the momentum conservation equations, which requires the pressure field to be corrected by,

$$
p=\widetilde{p}+\frac{\phi}{\Delta t}
$$

13. Finally, the solution procedure ends by checking if the end time is reached. If not, the procedure returns to step 3.

This new viscoelastic IB solver was implemented in the CFDEMcoupling framework, and its validation is performed with four benchmark case studies, which are presented in the next section. 


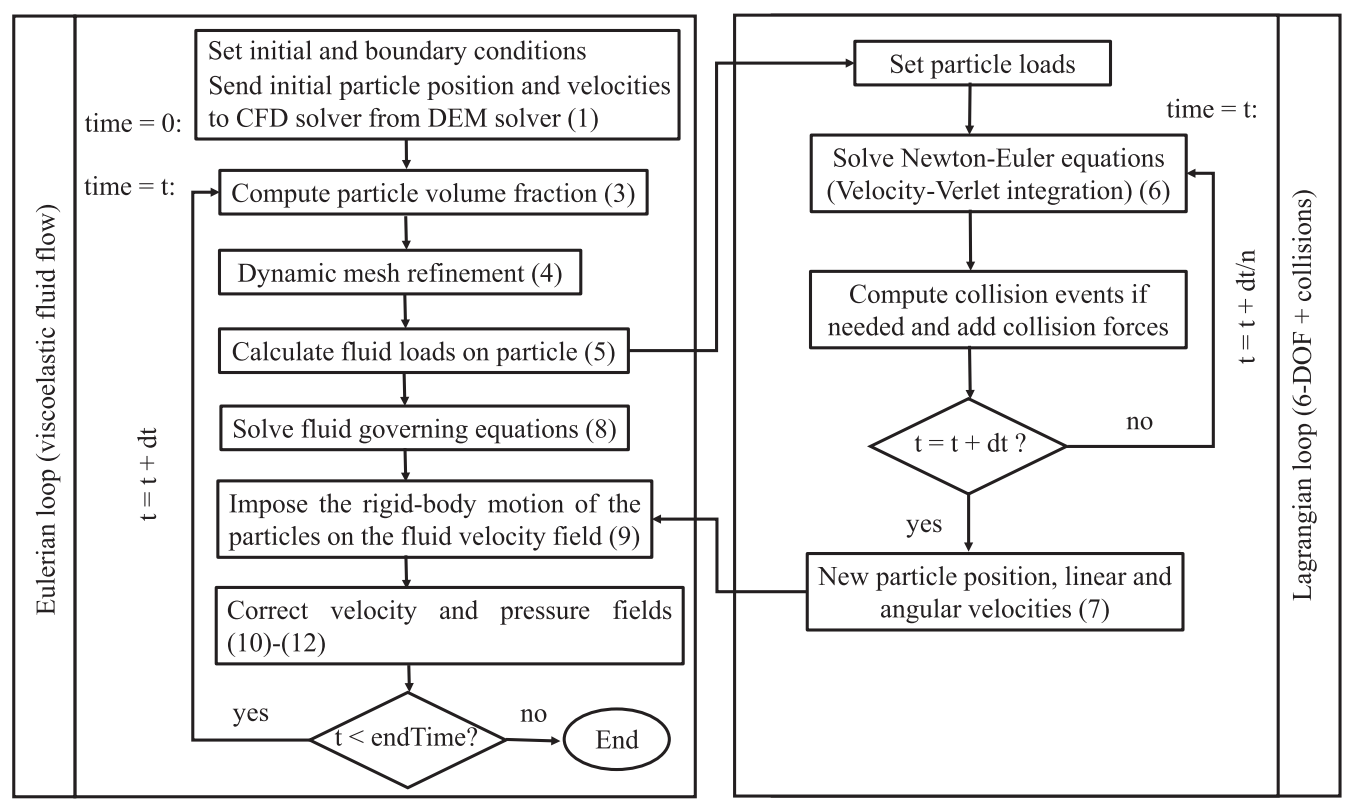

Fig. 1. Algorithm for the coupled CFD-DEM viscoelastic IB solver, where $t$ is time, $d t$ is the time-step size for the CFD solver and $d t / n$ is the equivalent time-step for the DEM solver.

\section{Validation case studies}

This section presents the validation of the newly-developed viscoelastic IB solver, by using it on several benchmark case studies that have already been presented in the scientific literature. The first case study is devoted to the sedimentation of a sphere in a rectangular duct containing a Newtonian fluid, which will verify the solver's capabilities to predict the steady-state sphere velocity accurately. Subsequently, the second case study describes the transient sedimentation dynamics of a sphere in several different viscoelastic fluids. This case study will allow us to test the implementation of the constitutive equations on the Eulerian fluid phase, and characterize the spatial and temporal orders of convergence of the solver. Additionally, high Weissenberg number simulations will be performed to verify the ability of the log-conformation approach to stabilize the numerical code. The third case study will verify the correctness of the 6-DOF code, on the Lagrangian loop, to compute the angular velocity of a sphere immersed in a steady shear flow of an Oldroyd-B fluid. Finally, the fourth integrated case study describes the cross-stream migration of a neutrally buoyant sphere in Poiseuille flow for both Newtonian and viscoelastic liquids. Here, the Segré-Silberberg effect [60] is reproduced for the Newtonian fluid and the influence of the retardation ratio on the particle equilibrium position is studied for the case of a Giesekus fluid.

\subsection{Case study 1: Sedimentation of a sphere in a Newtonian fluid}

Consider the settling motion of a sphere with velocity $U_{s}$ and radius $a$ as it falls along the centerline of a closed square box of width $W$ and height $H$ containing a Newtonian fluid (see Fig. 2). The box aspect ratio is fixed at $H / W=4$. No-slip conditions apply at all six bounding walls. This benchmark is designed to test the effect of changing the blockage ratio $(2 a / W)$ on the sphere settling velocity, when using different levels of maximum dynamic mesh refinement.

The dimensionless Reynolds number is set to $R e \equiv 2 \rho_{f} U_{0} a / \mu=0.36$, where $U_{0}$ is the unbounded terminal velocity, the so-called Stokes settling velocity [1], and $\mu$ is the dynamic viscosity of the fluid. The relative density of the sphere, given by a dimensionless weight or more simply by a density contrast, equals to $\rho_{s} / \rho_{f}=2.5$, where $\rho_{f}$ and $\rho_{s}$ are the fluid and sphere densities, respectively. The Newtonian calculations

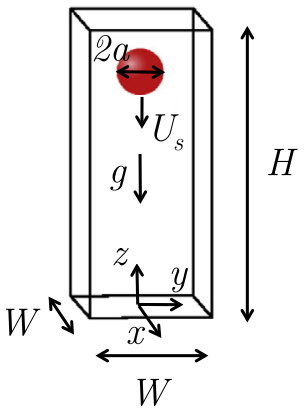

Fig. 2. Transient motion of a single-sphere falling through an initially quiescent viscous fluid confined in a square box of width $W$ and height $H$. The schematic diagram illustrates the computational domain including the coordinate system: $U_{s}$ is the downward velocity of the falling sphere of diameter $2 a$ and $g$ is the gravitational acceleration.

follow the same procedure illustrated in Fig. 1 with minor modifications on Eqs. (2), (15), (18) and (19) where the polymeric stress tensor $\tau_{P}$ is neglected. The Stokes settling velocity, $U_{0}$, expected for a sphere with radius $a$ in an unbounded domain $(a / W \rightarrow 0)$ containing a viscous Newtonian fluid, is calculated by equating the gravitational body force on the sphere with the viscous drag exerted on the sphere, which results in the following expression [1]

$U_{0}=\frac{(4 / 3) \pi a^{3}\left(\rho_{s}-\rho_{f}\right) g}{6 \pi \mu a}=\frac{2 a^{2}\left(\rho_{s}-\rho_{f}\right) g}{9 \mu}$

Because of the additional shear stresses introduced by the bounding walls of the box, the actual settling velocity $U_{s}$ in a box with finite blockage ratio $(2 a / W \neq 0)$ is expected to be much lower than $U_{0}$.

Fig. 3 shows the steady velocity of the sphere $U_{s}$ (monitored on the file with the particle velocity, which is written by the LIGGGHTS code with the print command) normalized by the Stokes velocity $U_{0}$ for different blockage ratios $2 a / W$. The best curve fit to the experimental data of Miyamura et al. [41] (solid line) is also shown for comparison purposes. The numerical results are computed using a uniform mesh with $20 \times 20 \times 80$ cells and with dynamic mesh refinement at the fluid-sphere interface. Two different maximum refinement levels are used, denoted 


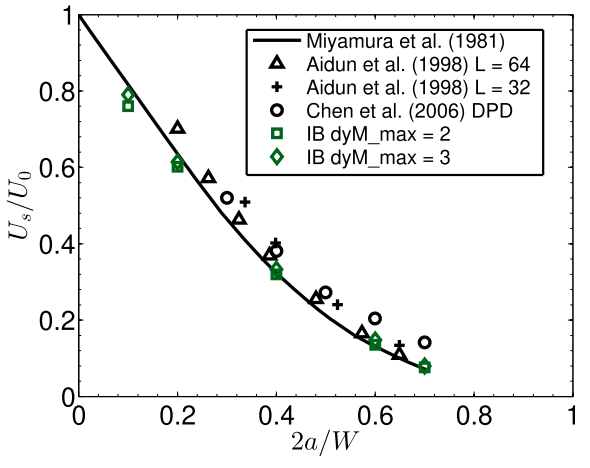

Fig. 3. Comparison between the steady-state velocity of a sphere falling in a closed container with blockage ratio $2 a / W$, filled with a Newtonian fluid, and the best curve fit to the experimental data of Miyamura et al. [41], the latticeBoltzmann simulation results of Aidun et al. [61] (with lattice $\mathrm{L}=64$ and $\mathrm{L}=$ 32 ), and the dissipative particle dynamic (DPD) simulation results of Chen et al. [9].


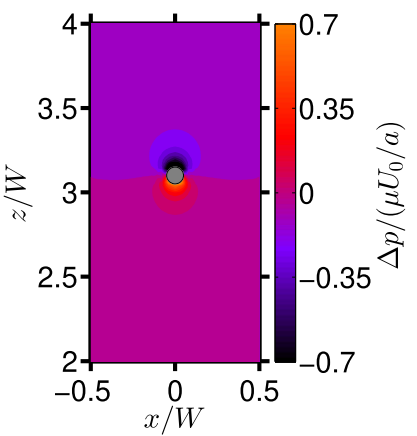

(a)

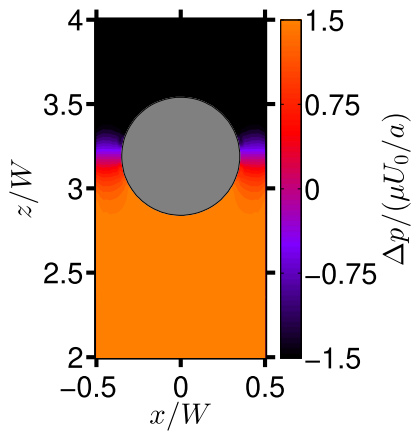

(b)
Fig. 4. Normalized velocity (top) and pressure difference $(\Delta p=p-p(x / W=$ $0.5, z / W=z_{\text {sphere center }}$ )) (bottom) contours for the sedimentation of a sphere in a Newtonian fluid settling along the centerline of a square duct with blockage ratio (a) $2 a / W=0.1$ and (b) $2 a / W=0.7$.

by dyM_max, for each blockage ratio tested, with typical values equal to two or three. Additionally, the lattice-Boltzmann simulation results of Aidun et al. [61], which include a fine lattice $(L=64)$ and a coarser lattice $(\mathrm{L}=32)$, and the dissipative particle dynamic (DPD) simulation results of Chen et al. [9] are included for comparison purposes. As shown in Fig. 3 the numerical results obtained with both of the maximum mesh refinement levels, are in good agreement with previous experiment [41], lattice-Boltzmann [61] and DPD [9] simulation results, for all the blockage ratios considered. Additionally, in Fig. 4 we show the normalized velocity (top) and pressure (bottom) contours for blockage ratios $2 a / W=0.1$ and $2 a / W=0.7$. As can be seen by the velocity contours, when increasing the blockage ratio the restrictive effect of the walls on the steady settling velocity of the particle increases markedly. The no-slip boundary condition at the outer wall decreases the particle settling velocity and increases the local fluid velocity in the 'nip'

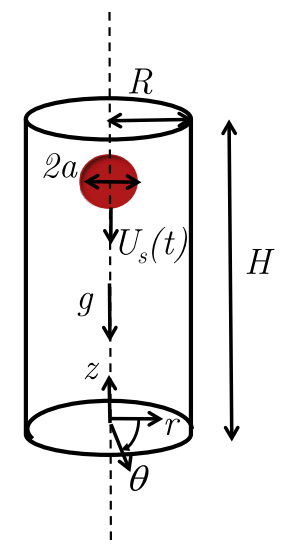

Fig. 5. Geometry and cylindrical coordinate system for the transient motion of a single sphere of radius $a$ falling through an initially quiescent viscoelastic fluid confined in a tube of radius $R$ and height $H$. Here $U_{s}(t)$ is the time- varying downward velocity of the falling sphere after it is released from rest at $t=0$ and $g$ is the gravitational acceleration.

region near the equator of the sphere. This also means that as the fluid is squeezed between the particle and the walls, there is an increase in the total pressure difference between the front and rear stagnation points, as shown by the corresponding pressure contours.

\subsection{Case study 2: Sedimentation of a sphere in viscoelastic fluids}

The first case study employed to validate the newly implemented viscoelastic IB algorithm was the transient settling motion of a sphere with velocity $U_{s}(t)$ and radius $a$, as it falls along the centerline of a cylindrical tube of radius $R$ containing a viscoelastic fluid (see Fig. 5). No-slip conditions were considered at all bounding walls. If the sphere is released from rest, then the viscoelastic nature of the fluid results in a temporal retardation of the viscous drag force counteracting the gravitational acceleration of the solid body, and theoretical analyses with linear viscoelastic models predict an initial velocity overshoot in the motion of the sphere [62]. As the motion of the sphere approaches steady state, viscoelasticity and the presence of the bounding walls lead to modifications in the velocity field in the surrounding fluid, and the steady-state velocity of the sphere may be substantially different from that observed in a Newtonian fluid.

The geometry for this case study follows the one used by Rajagopalan et al. [42]. In this experiment, a long plexiglass cylinder of height $H=90 \mathrm{~cm}$ and internal radius $R=5.23 \mathrm{~cm}$ is filled with a Boger fluid, and its axis is carefully aligned to be parallel with the gravity axis. The spheres used in the experiments were commercially available ball bearings of diameter $2 a=2.54 \mathrm{~cm}$. This way, the radius ratio is fixed at $\mathcal{K}=a / R \approx 0.243$. The ceramic sphere has a density of $\rho_{s}=3.84 \mathrm{~g} / \mathrm{cm}^{3}$ and the fluid density is $\rho_{f}=0.895 \mathrm{~g} / \mathrm{cm}^{3}$, which gives the relative density of the sphere equal to $\rho_{s} / \rho_{f} \approx 4.29$.

With respect to the fluid rheology, the same parameters as in Rajagopalan et al. [42] were used, where first the zero-shear-rate properties of the viscoelastic test fluid, determined using a cone-and-plate rheometer at a reference temperature $T_{0}=25{ }^{\circ} \mathrm{C}$, are fixed as $\eta_{S}=$ 8.12 Pa.s, $\eta_{0}=\eta_{S}+\eta_{P}=13.76$ Pa.s and $\Psi_{10}=8.96$ Pa.s $^{2}$. The mean relaxation time is then given by $\lambda=\Psi_{10} /\left[2\left(\eta_{0}-\eta_{S}\right)\right] \approx 0.794 \mathrm{~s}$ and the retardation ratio by $\beta=\eta_{S} / \eta_{0} \approx 0.59$.

The steady Newtonian settling velocity $U_{N}$, expected for a sphere with radius $a$ settling along the centerline of a tube of radius $R$ filled with a viscous Newtonian solvent of equivalent viscosity $\eta_{0}$, is given by a modification to Eq. (25) incorporating the Faxen wall correction factor $K_{N}(\mathcal{K})[63]:$

$U_{N}=\frac{(4 / 3) \pi a^{3}\left(\rho_{s}-\rho_{f}\right) g}{6 \pi \eta_{0} a K_{N}(\mathcal{K})}=\frac{2 a^{2}\left(\rho_{s}-\rho_{f}\right) g}{9 \eta_{0} K_{N}(\mathcal{K})}$ 


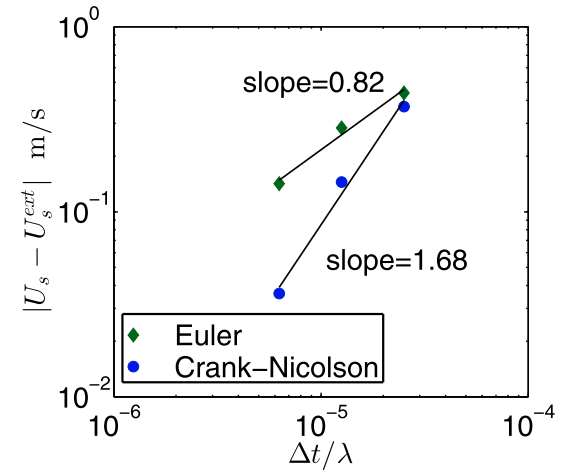

Fig. 6. Numerical error as a function of the computational time-step at $t / \lambda=0.2$ for case study 2 . The solid lines represent linear fits to the numerical points on a log-log scale and the slope gives the apparent order of convergence for each scheme.

where $g$ is the gravitational acceleration, $\mathcal{K}=a / R$ and $K_{N}(\mathcal{K})$ describes the reduction in the velocity resulting from the presence of walls. For $\mathcal{K}=0.243$, the correction factor is $K_{N}=1.93$ [63], which results in a settling velocity of $U_{N} \approx 3.91 \mathrm{~cm} / \mathrm{s}$.

The relevant dimensionless quantities measuring the importance of viscous, inertial, and elastic effects are defined using nominal Reynolds and Weissenberg numbers in terms of the settling velocity $U_{N}$ expected for such a sphere as

$$
R e=\frac{2 a \rho_{f} U_{N}}{\eta_{0}}
$$

and

$W i=\frac{\lambda U_{N}}{a}$

respectively. This way, the nominal values of the $R e$ and Wi numbers estimated a priori using the settling velocity $U_{N}$, are $R e \approx 0.064$ and $W i \approx 2.45$. To establish the temporal and spatial accuracy of the viscoelastic IB code, the viscoelastic fluid is described with a single-mode FENE-CR model, using a finite extensibility of the polymer molecules equal to $L^{2}=10$. The reason to choose this model and the value of $L^{2}$ for these studies is because the steady-state particle velocity is reached faster than in other viscoelastic models (see Fig. 4 in [42]). Additionally, the meshes employed on the temporal and spatial sensitivity studies in this section do not include any dynamic mesh refinement level on the sphere-fluid interface.

Firstly, the order of convergence for the discretization of the rate of change term was evaluated for two different discretization schemes, Euler and Crank-Nicolson, with three different time-step sizes, $\Delta t / \lambda=$ $\{2.5,1.25,0.625\} \times 10^{-5}$. The numerical error, given by the absolute difference between the sphere velocity $\left(U_{s}\right)$ and the extrapolated sphere velocity $\left(U_{s}^{\text {ext }}\right.$, obtained from the Richardson extrapolation technique [64] using the three time-step refinement levels stated above), as a function of the time-step at a fixed time of $t / \lambda=0.2$ is shown in Fig. 6 . The mesh used for the time-step refinement study has 9 cells per particle diameter $(\Delta r / a=2 / 9)$, with a total of 347200 mesh cells. The results illustrated in Fig. 6 confirm that the numerical method retains, approximately, the theoretical order of accuracy of the time schemes used: the Crank-Nicolson scheme is second-order accurate, while the Euler scheme is only first-order accurate.

Additionally, the spatial order of convergence of the numerical method was assessed using two different advection discretization schemes, Upwind (UDS) and CUBISTA [65], and four different mesh refinement levels, $\Delta r / a=\{1 / 2,1 / 3,2 / 9,4 / 27\}$, labeled M1 to M4, respectively. Table 1 gives the total number of cells and degrees of freedom (DOF) corresponding to each one of the mesh refinement levels employed. Notice that, for the DOF calculation, there exist 10 dependent fluid variables ( 3 velocity components, pressure and 6 extra-stress
Table 1

Total number of mesh cells and degrees of freedom (DOF) for the meshes employed in the spatial order of convergence study.

\begin{tabular}{lllll}
\hline Mesh & $\Delta r / a$ & $\begin{array}{l}\text { Total number } \\
\text { of mesh cells }\end{array}$ & $\begin{array}{l}\text { Estimated number of cells } \\
\text { covered by solid fraction }\end{array}$ & DOF \\
\hline M1 & $1 / 2$ & 32850 & 33 & 328698 \\
M2 & $1 / 3$ & 105135 & 113 & 1052028 \\
M3 & $2 / 9$ & 347200 & 380 & 3474280 \\
M4 & $4 / 27$ & 1196338 & 1285 & 11971090 \\
\hline
\end{tabular}
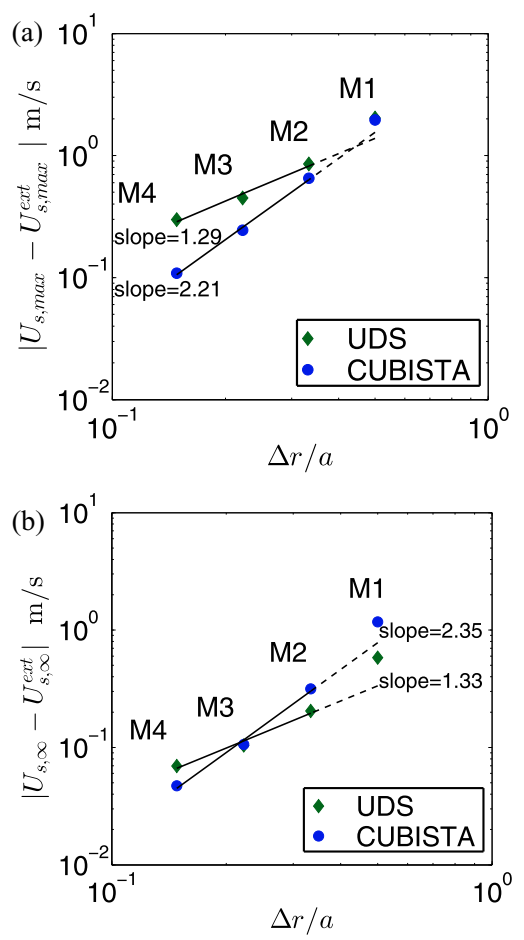

Fig. 7. Error as a function of the mesh resolution, for (a) maximum and (b) steady-state sphere velocities for case study 2 . The solid lines represent linear fits to the numerical values (M2-M3-M4) on a log-log scale and the corresponding slope gives the convergence order for each scheme. The dashed lines are an extrapolation of the linear fit to M1.

components) in all domain cells, plus 6 dependent particle variables (3 translational velocity components and 3 angular velocity components) in the cells covered by the solid fraction (which were estimated by the ratio of the particle and a cubic cell volume, using the respective value of $\Delta r / a$ ). The time-step level used to compute the spatial order of convergence of the algorithm was $\Delta t / \lambda=1.25 \times 10^{-5}$. The order of convergence is computed for both the maximum, $U_{s, \max }$, and steadystate, $U_{s, \infty}$, sphere velocities. The results are plotted in Fig. 7 , where the upwind scheme is found to converge approximately with order of 1.29 and 1.33, and the CUBISTA scheme converges with order of 2.21 and 2.35, using the maximum and steady-state velocities, respectively. The results obtained show that the theoretical order of accuracy of the advective discretization schemes used (UDS order 1 and CUBISTA order 2) are approximately retained by the new viscoelastic IB algorithm.

The dimensionless velocity of the sphere $U_{s}(t) / U_{N}$, computed numerically for the viscoelastic fluids described by the single-mode OldroydB $\left(L^{2} \rightarrow \infty\right)$ and FENE-CR constitutive models, is compared with the results obtained by Rajagopalan et al. [42] with a FEM simulation, as shown in Fig. 8 (see also the movies in the supplementary material). The numerical results are computed using a mesh similar to M3 $(\Delta r / a=2 / 9)$ and a time-step level of $\Delta t / \lambda=1.25 \times 10^{-5}$. Additionally, a dynamic mesh refinement level equal to two is used in order to improve the accuracy of the numerical results obtained. It can be seen that 
(a)

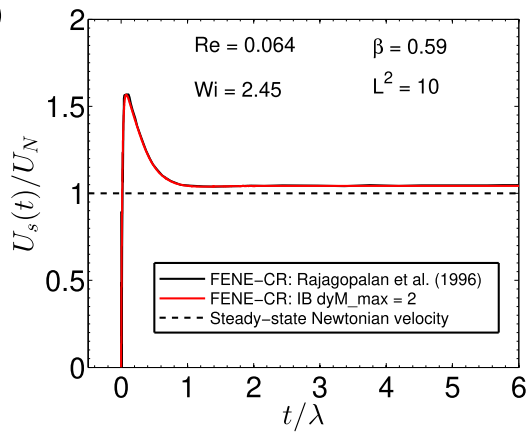

(b)

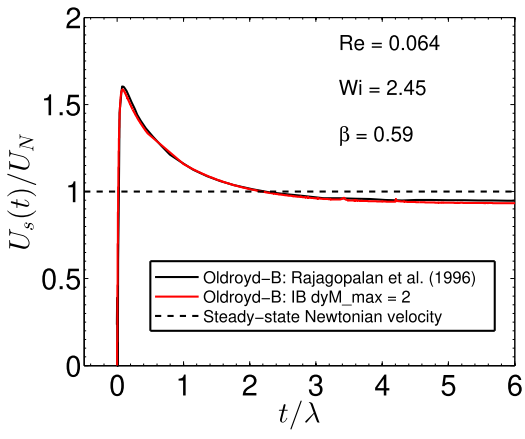

Fig. 8. Predicted initial transient motion of a sphere through a (a) FENE-CR and (b) Oldroyd-B fluid, for case study 2 (see also the movies in the supplementary material). Solid black lines represent the results of Rajagopalan et al. [42] and red lines represent numerical results obtained with maximum dynamic mesh refinement level of two. The dashed black line represents the Newtonian steadystate velocity. (For interpretation of the references to colour in this figure legend, the reader is referred to the web version of this article.)

viscoelasticity affects both the transient and steady state settling velocities of the sphere. At steady state the finite extensibility of the fluid controls whether the sphere falls faster than the Newtonian value $U_{N}$ given by Eq. (26) (e.g. for $L^{2}=10$ ) or more slowly (e.g. for $L^{2} \rightarrow \infty$, i.e., the Oldroyd-B model). The most interesting behavior occurs at intermediate times $(t / \lambda \approx 0.1)$, where the quasi-linear Oldroyd-B and FENE-CR models predict a velocity overshoot and the maximum velocity is approximately $U_{\max } / U_{N} \approx \eta_{0} / \eta_{S}=1.69$. As can be seen in Fig. 8 the numerical results obtained by the newly-developed solver are very close to the ones presented by Rajagopalan et al. [42], which is an evidence of the correct implementation of the new viscoelastic IB algorithm.

For this second case study, we also tested the Giesekus viscoelastic model, with mobility parameter $\alpha=0.2$ (representative of shearthinning viscoelastic behavior), to describe the fluid rheology. The particle settling trajectory was computed for $W i=2.45$ and $W i=10$, and for two different values of the retardation ratio $\beta=\eta_{S} / \eta_{0}=0.1$ and $\beta=0.59$ (see Fig. 9). For the high Wi number case $(W i=10)$ the log-conformation approach is used to compute the polymeric extra-stress tensor components, which improves the numerical stability of the developed algorithm [48]. As shown in Fig. 9 (see also the movies in the supplementary material), for the same retardation ratio values, increasing the Weissenberg number increases the steady-state particle settling velocity substantially above $U_{N}$, due to the onset of shear thinning. Additionally, the decrease of the retardation ratio from $\beta=0.59$ to $\beta=0.1$ induces a pronounced secondary undershoot in the particle settling velocity due to the reduction in the level of damping provided by the viscous Newtonian solvent. This effect is present at both values of the Weissenberg number but enhanced at $W i=2.45$, before the effects of shear thinning become totally dominant, at $W i \gg 1$.

To understand the above behavior, we present the fluid streamlines in Fig. 10 for $W i=2.45, R e=0.064$ and $\beta=0.1$, at four characteristic moments in the time series, $t / \lambda=\{0.06,0.14,0.5,2.5\}$. At the beginning, $t / \lambda=0.06$, the flow around the sphere is fore/aft symmetric

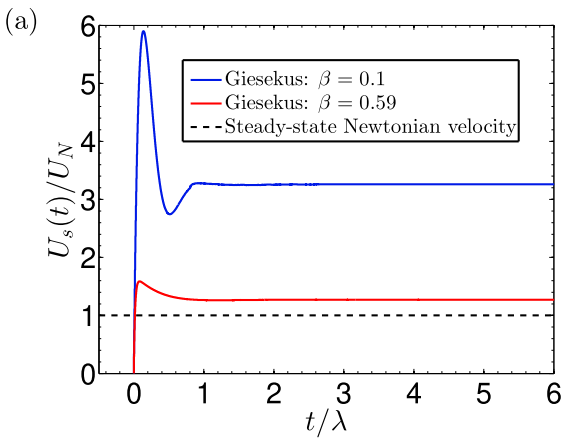

(b)

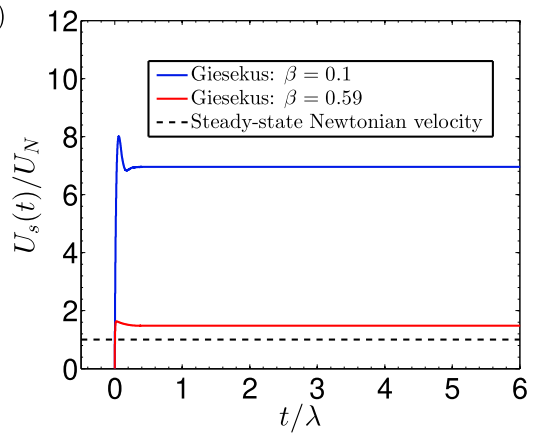

Fig. 9. Predicted initial transient motion of a sphere through a Giesekus fluid ( $\alpha=0.2$ ) for case study 2 (see also the movies in the supplementary material). Effect of the Weissenberg number, (a) $W i=2.45$ and (b) $W i=10$, and retardation ratio ( $\beta=0.1$ and $\beta=0.59$ ) on the sphere settling velocity at $R e=0.064$.
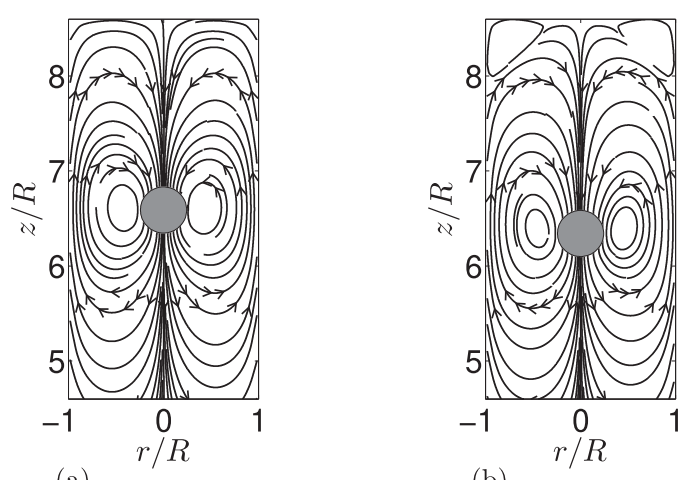

(b)

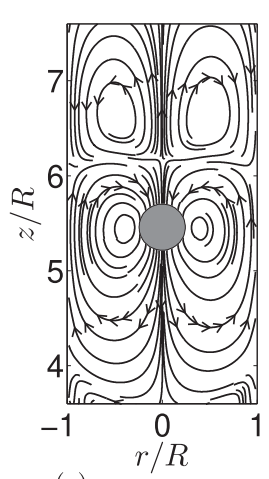

(c)

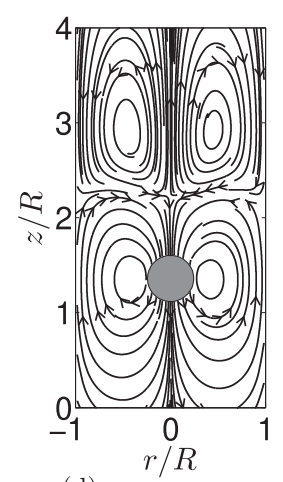

(d)

Fig. 10. Streamlines for the case study 2 with Giesekus fluid model with $W i=$ 2.45, $R e=0.064$ and $\beta=0.1$ at different moments in the time series: (a) $t / \lambda=$ 0.06 , (b) $t / \lambda=0.14$, (c) $t / \lambda=0.5$ and (d) $t / \lambda=2.5$. 

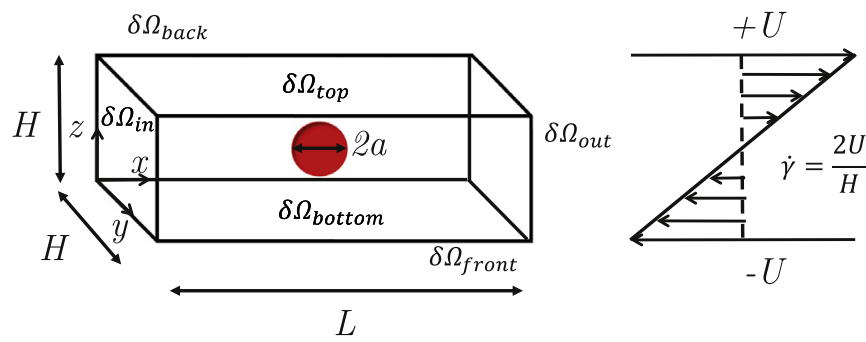

Fig. 11. Geometry used for the rotation of a sphere of diameter $2 a$ in a viscoelastic liquid subjected to shear flow.

and Fig 10(a) shows that the fluid in the wake of the sphere moves with a downward velocity (positive wake). At the time of the maximum overshoot in $U_{s}(t / \lambda=0.14)$, Fig 10(b) shows a strongly fore-and-aft asymmetric flow with the center of the recirculation displaced upwards substantially beyond the equator of the sphere. Next, at the end of the deceleration period that follows the overshoot, $t / \lambda=0.5$, Fig 10 (c) shows that a new flow region in the wake of the sphere with upward velocity (negative wake) has developed. This negative wake is a stable structure that remains intact after steady state has been reached, $t / \lambda=2.5$, as shown in Fig 10(d) and is consistent with previous computations performed with custom-developed codes $[24,32,66]$.

\subsection{Case study 3: Rotation of a sphere in a fluid subjected to shear flow}

The problem of the dynamics of a single sphere immersed in a linear flow field imposed at infinity, in the absence of both fluid and particle inertia, was first addressed by Einstein [67] for the case of a Newtonian suspending medium. Under steady shear flow, the sphere translates in the flow direction at steady velocity while also rotating around the vorticity axis. Thus, in a frame translating with the sphere center, the sphere just rotates in time with a constant angular velocity $\omega$. Einstein [67] demonstrated that, under no-slip boundary conditions at the particle surface, the rotation rate at steady state is given by $\omega=\dot{\gamma} / 2, \dot{\gamma}$ being the externally imposed shear rate. This simple result stems from a torque balance at the sphere surface, whereby the torque on the sphere is only due to the flow field (the so called torque-free condition) [68]. The rotation speed of the particle is independent of the particle radius and viscosity of the fluid.

In this test, the 3D flow problem is solved with the new viscoelastic IB code in a rectangular box (see Fig. 11) with height $H$ and length $L=3.75 H$, containing a single sphere of diameter $2 a$ at the center of the box, with $2 a / H=0.25$. The boundary conditions applied are as follows: periodic boundary condition on $\delta \Omega_{\text {in }}, \delta \Omega_{\text {out }}, \delta \Omega_{\text {back }}$ and $\delta \Omega_{\text {front }}$ for all fields, zero gradient value on $\delta \Omega_{\text {top }}$ and $\delta \Omega_{\text {bottom }}$ for pressure, and fixed value uniform profile for velocity, with velocity vectors given by ( $U$, $0,0)$ and $(-U, 0,0)$, respectively, where $U$ is the imposed velocity. The imposed shear rate is then $\dot{\gamma}=\frac{2 U}{H}$. In this case study, the ratio of inertia and viscous forces, defined by the Reynolds number of the fluid in the box, is given by $R e_{H} \equiv \frac{2 \rho_{f} U H}{\eta_{0}}=8$, where $\eta_{0}$ is the total fluid viscosity.

Following Snijkers et al. [43], the ratio $\omega / \dot{\gamma}$ is plotted as a function of the Weissenberg number for an Oldroyd-B fluid, considering the retardation ratio and Weissenberg number to be $\beta=0.5$ and $W i=\lambda \dot{\gamma}=$ $\{0.25,0.5,1,2\}$, respectively. Here, the numerical results are obtained using two different calculation procedures, one using a fixed-mesh approach with a newly developed boundary condition in our previous study [69], and the other using the viscoelastic IB code. For the fixedmesh approach the fluid forces are integrated on the sphere surface and used to compute the torque and angular velocity generated, which is then imposed as a boundary condition on the sphere surface and monitored along the simulation time until it reaches the steady-state value. The mesh used on the fixed-mesh approach was generated by the snappyHexMesh utility of the OpenFOAM computational library, which is

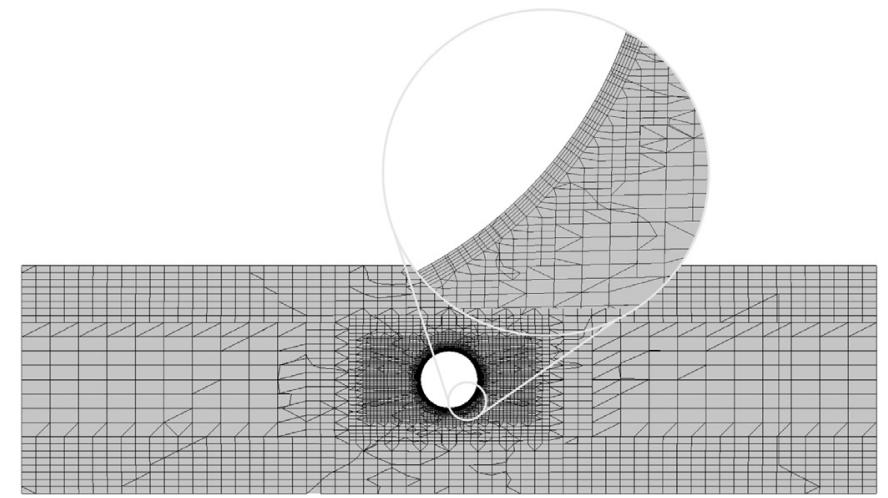

Fig. 12. Mesh generated by the snappyHexMesh utility on the center plane of the shear flow geometry.

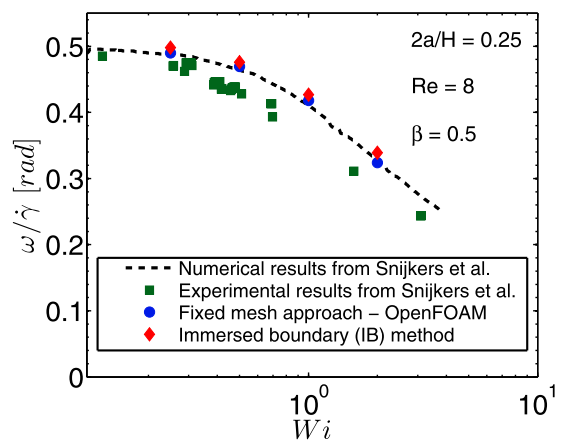

Fig. 13. Sphere angular velocity $\omega / \dot{\gamma}$ as a function of Weissenberg number in a pure shear flow, computed by a viscoelastic fixed mesh and IB codes, and comparison with Snijker et al.'s [43] experimental and numerical results.

capable of creating a local surface refinement by setting a predefined level of refinement ( 5 in this study), and a smooth transition between a predefined number of layers ( 6 in this study) with an expansion ratio between each layer (1.05 in this study). The mesh generated has a total of 1251628 cells and the time-step used in the simulations was $d t=10^{-8}$ s. Fig. 12 shows the mesh, in the central plane, generated by the snappyHexMesh utility and the zoom therein enhances the layers near the sphere surface. In the second approach, where the viscoelastic IB code is used, the mesh is generated with 16 cells per sphere diameter, which gives a total of 983040 cells, and the Eulerian and Lagrangian time-steps used were $d t_{E u l}=10^{-5} \mathrm{~s}$ and $d t_{\text {Lag }}=10^{-6} \mathrm{~s}$, respectively. In Fig. 13 we show the comparison between the results obtained by Snijkers et al. [43] (experimental and numerical) and those obtained by the two approaches described above. As can be seen both methods are able to accurately reproduce the results from Snijkers et al. [43] for an Oldroyd-B liquid.

Fig. 14 shows a single iso-contour of the extra-stress tensor trace $\left(\operatorname{tr}\left(\tau_{P}\right)\right)$, colored by the value of the normal extra-stress tensor component $\tau_{x x}$ on that contour, for the different Weissenberg numbers tested. To plot these structures we choose the isoline at which the rear and front wakes have merged with the north and south poles of the particle. The results obtained show that increasing the Weissenberg number induces bigger structures in the wakes, but smaller ones near the poles. These large extended elastic wakes or 'wings' act to retard the rotation of the sphere [10].

\subsection{Case study 4: Cross-stream migration of a neutrally buoyant sphere in Poiseuille flow}

Neutrally buoyant spheres immersed in a steady Poiseuille flow in a tube of radius $R$ are subject to lift forces that drive the spheres either 




(a) Isoline at $\operatorname{tr}\left(\boldsymbol{\tau}_{P}\right) / \eta_{0} \dot{\gamma}=0.34$

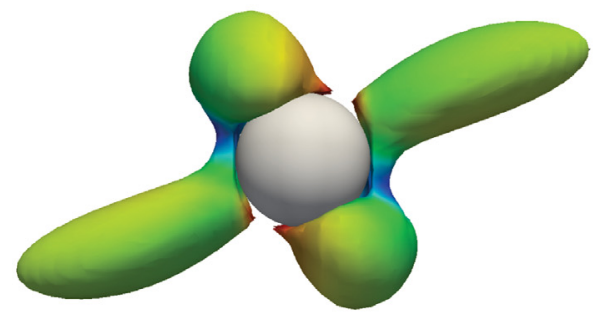

(b) Isoline at $\operatorname{tr}\left(\boldsymbol{\tau}_{P}\right) / \eta_{0} \dot{\gamma}=0.59$
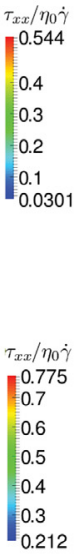

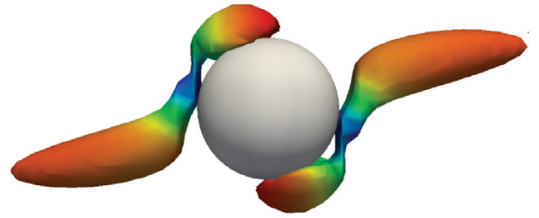

(c) Isoline at $\operatorname{tr}\left(\boldsymbol{\tau}_{P}\right) / \eta_{0} \dot{\gamma}=1.5$
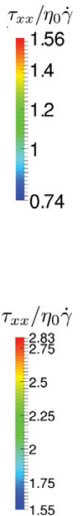

(d) Isoline at $\operatorname{tr}\left(\boldsymbol{\tau}_{P}\right) / \eta_{0} \dot{\gamma}=2.8$


$\omega / \dot{\gamma}=0.428$; and (d) $W i=2, \omega / \dot{\gamma}=0.339$.

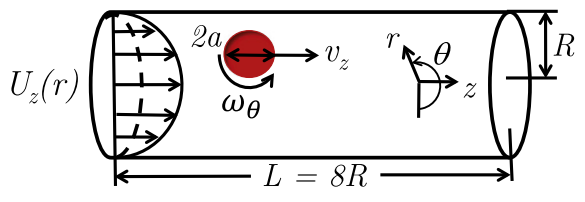

Fig. 15. Schematic illustrating the computational domain (in cylindrical coordinates) for case study $4: U_{z}(r)$ is the inlet flow velocity, $\omega_{\theta}$ is the angular velocity of the sphere (of radius $a$ ) in the $\theta$ direction, $v_{z}$ is the sphere axial velocity, and $R$ is the tube radius.

(a) away from the wall towards steady radial positions $r_{s s}$, nearer the tube centerline, or (b) alternatively towards the wall, for initial release radii smaller than a threshold value. For Poiseuille flow of a Newtonian fluid, the equilibrium position, at which the net lift force on a sphere is zero, is located between the tube centerline and the wall, corresponding to $r_{s s} / R \approx 0.6$. This phenomenon is known as the Segré and Silberberg [60] or "tubular pinch" effect.

This Segré-Silberberg effect is reproduced here for an additional validation of the developed solver by simulating the transport of a single neutrally buoyant sphere in a circular tube (see Fig. 15). The computational setup is the same as that used in previous numerical studies $([35,44])$ of the Segré and Silberberg [60] effect: The tube has a radius of $R=2.5 \mathrm{~mm}$ and a length of $8 R$, the sphere radius is $a=0.15 R$, the fluid kinematic viscosity $(v)$ is $1 \mathrm{cSt}$, the density of the fluid and sphere are both $1000 \mathrm{~kg} / \mathrm{m}^{3}$, the mean fluid velocity at the inlet is $10 \mathrm{~mm} / \mathrm{s}$ (corresponding to a tube Reynolds number of $R e=50$ ), and the initial radial positions for the sphere are $r_{i} / R=0.2$ and $r_{i} / R=0.75$. As in the previous numerical studies $[35,44]$, a periodic boundary condition is applied at the inlet and outlet for all fields, and a no-slip velocity/zero-gradient pressure boundary condition is specified on the tube wall.

As shown in Fig. 16 (see also the movies in the supplementary material), the results obtained with the new IB code for the cross-stream migration of a neutrally buoyant sphere in a Newtonian fluid undergoing steady Poiseuille flow, compare well with the theoretical equilibrium position of $r_{s s} / R \approx 0.6$ for both of the particle initial release positions.

Next, the newly-developed viscoelastic IB code is used to compute the cross-stream migration of a sphere immersed in a Giesekus fluid, with mobility parameter $\alpha=0.2$. The particle equilibrium position is obtained for $W i=1, R e=50$ and three different retardation ratios $\beta=\{0.1,0.5,0.9\}$. As shown in Fig. 17, for the particle released near the wall $\left(r_{i} / R=0.75\right)$, increasing the retardation ratio moves the particle to an equilibrium position of approximately $r_{s s} / R=0.65$. For the case where the particle is released near the tube center $\left(r_{i} / R=0.2\right)$,

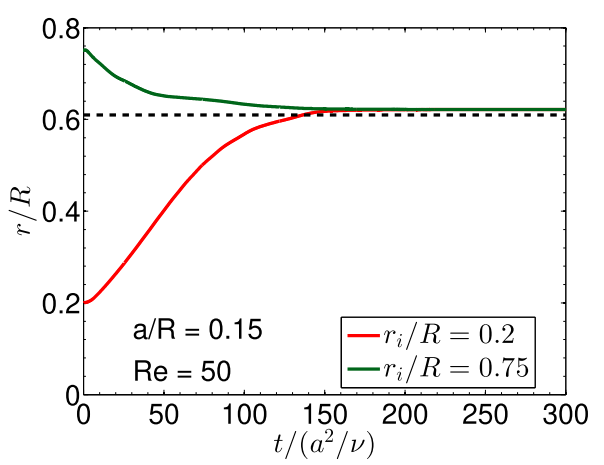

Fig. 16. Evolution of the radial position for a sphere in a Newtonian fluid undergoing steady axisymmetric Poiseuille flow released from two different starting positions in the pipe, $r_{i} / R=0.2$ and $r_{i} / R=0.75$ (see also the movies in the supplementary material). The dashed black line represents the theoretical Segré-Silberberg equilibrium position.

increasing the retardation ratio is found to have a non-monotonic effect on the particle equilibrium position, that is, for the low and high values of $\beta$ the particle migrates to $r_{s s} / R \approx 0.6$, but for the intermediate value of $\beta$, it migrates to $r_{s s} / R \approx 0.65$. As the retardation ratio $\beta$ is increased, the migration velocity becomes progressively higher. These results indicate that incorporating the effects of the fluid viscoelasticity has a complex transient effect on the equilibrium position of the sphere, which requires further investigation that will be considered in future works.

\section{Particle alignment in viscoelastic fluids}

As first reported by Michele et al. [70] rigid particles suspended in viscoelastic fluids under shear can aggregate into string-like structures aligned in the flow direction. The driving force for this phenomenon was attributed to the effect of the normal stress differences generated by the viscoelastic shear flow on the rigid surfaces of the particles. Experiments by Scirocco et al. [71] and Pasquino et al. [72] have shown that the alignment effect can be substantially enhanced (resulting in more rapid shear-driven alignment) by the presence of confining rigid walls. Recently, experimental work carried out by Loon et al. [73] showed particle alignment in a shear-thinning fluid without significant normal stress differences. Additionally, aiming to provide further insights on the phenomenon of particle alignment, Jaensson et al. [74] conducted, for the first time, direct 3D numerical simulations of the alignment of two and three rigid, non-Brownian spherical particles in an unbounded 
(a)

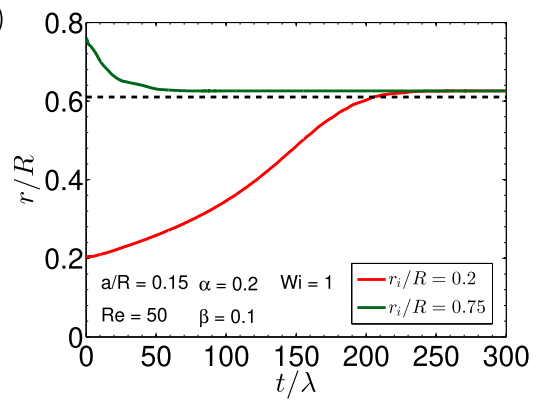

(b)



(c)

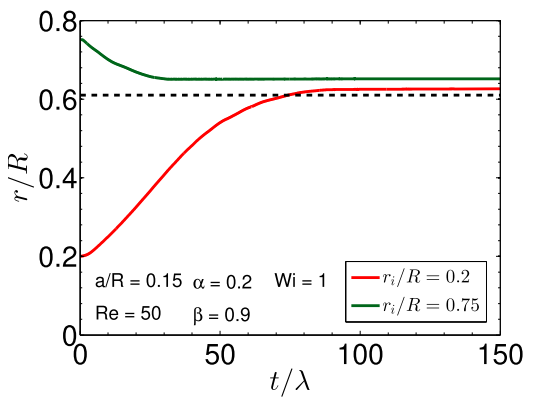

Fig. 17. Evolution of the radial position for a sphere in a Giesekus fluid undergoing steady Poiseuille flow at $W i=1, R e=50$ released from two different starting positions in the pipe, $r_{i} / R=0.2$ and $r_{i} / R=0.75$, for retardation ratio (a) $\beta=0.1$, (b) $\beta=0.5$ and (c) $\beta=0.9$.

viscoelastic shear flow. The effects of elasticity, shear thinning and the ratio between the solvent viscosity and zero-shear viscosity were investigated. They concluded that the action of normal stress differences is essential for particle alignment to occur, although it is also strongly enhanced by shear thinning. However, they did not consider the effects of rigid channel boundaries in their simulations.

To illustrate the capabilities of our new IBM viscoelastic solver, we have conducted a preliminary set of numerical simulations which demonstrate the effect of the blockage ratio $(2 a / H$ where $a$ is the particle radius and $H$ is the channel height) on particle alignment in viscoelastic fluids under steady planar shear flow. This study is expected to aid in unraveling complex phenomena which are still not completely understood, such as the particle alignment and migration in highly-elastic shear-thinning fluids in confined shear flow. However, a complete study of the effect of the channel gap size and fluid rheology on the particle alignment will be the subject of additional study in future works.

We consider three non-Brownian spherical particles in a steady planar shear flow, as depicted in Fig. 18. Regarding the size of the periodic domain we have considered two different cases: for the first case, the channel length $(L)$, width $(W)$, and height $(H)$ were set, respectively, to $40 a, 10 a$ and $16 a$, to ensure minimal boundary effects; and for the second case, $L, W$, and $H$ were set, respectively, to $20 a, 10 a$ and $8 a$, to evaluate the effects of reducing the gap size on the particle alignment rate.

Simulations have been performed to investigate whether a string is formed by three particles, where the central particle is placed at the

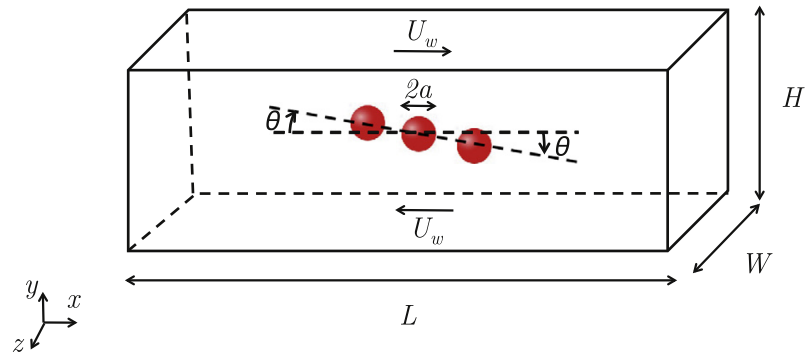

Fig. 18. Rigid spheres suspended in viscoelastic fluids under steady planar shear flow. The rectangular domain is bounded in the $y$-direction and periodicity is assumed at the boundaries normal to both $x$ and $z$ directions. The origin is located at the centroid of the domain, and $\theta$ is the angle between the $x$-axis and the line connecting the centers of the spheres.

Table 2

Computed and extrapolated steady-state distance and angular velocity, and relative errors obtained for each refinement level employed (dyM_max). The total number of mesh cells are approximations, since the mesh changes during the simulation (except for mesh M1 where dyM_max $=0$ ).

\begin{tabular}{llcllll}
\hline Mesh & dyM_max & $\begin{array}{l}\text { Total number } \\
\text { of mesh cells }\end{array}$ & $d_{f} / a$ & $\begin{array}{l}\text { Relative } \\
\text { error (\%) }\end{array}$ & $\left(\omega_{z}\right)_{f} / \dot{\gamma}$ & $\begin{array}{l}\text { Relative } \\
\text { error (\%) }\end{array}$ \\
\hline M1 & 0 & 12800 & - & - & 0.259 & 18.8 \\
M2 & 1 & $\sim 20000$ & 2.001 & 3.9 & 0.186 & 14.7 \\
M3 & 2 & $\sim 45000$ & 2.062 & 1.0 & 0.210 & 3.7 \\
Extrapolated & $\infty$ & - & 2.082 & - & 0.218 & - \\
\hline
\end{tabular}

origin (located at the centroid of the domain), and the outer particles are placed at an angle of $\theta=\pi / 18 \mathrm{rad}$, with an initial distance between the center points of the central and outer particles of $3 a$.

Due to the movement of the rigid top and bottom walls, a shear flow with an average shear rate of $\dot{\gamma}=2 U_{w} / H$ is imposed, where $U_{w}$ denotes the magnitude of the velocity of the walls.

When the particles come into contact, the non-linear elastic HertzMindlin contact model is used [52]. This model computes the frictional force between two particles considering the normal and tangential components, both comprising spring and damping components. To enforce a rigid behavior of the particles the damping terms were canceled by using a coefficient of restitution equal to one. The numerical case studies were performed with three constitutive models, Newtonian, Oldroyd-B and Giesekus, in order to consider, respectively, inelastic, viscoelastic constant shear viscosity and viscoelastic shear-thinning behaviors.

The relevant dimensionless numbers for this case study are the Reynolds number, defined by $R e=2 \rho U_{w} H / \eta_{0}$, the Weissenberg number, defined by $W i=\lambda \dot{\gamma}$, the mobility parameter $\alpha$ (which controls the shear-thinning behavior of the Giesekus fluid), the retardation ratio, denoted by $\beta=\eta_{S} / \eta_{0}$, and the blockage ratio $2 a / H$. For the runs presented here, some of the above dimensionless numbers were fixed, namely: $R e=0.1$ (representative of creeping flow conditions), $W i=3, \alpha=0.1$ and $\beta=0.1$.

For problems like the one presented in this section, where contact/collision occurs, the size of the mesh cells in the gap between the particles is very important. Thus, we start by investigating the mesh convergence using the Giesekus fluid model in the smaller domain (blockage ratio $2 a / H=1 / 4$ ), with three different meshes, labeled M1 to M3, which have maximum dynamic mesh refinement levels of dyM_max $=\{0,1,2\}$, respectively (see Table 2). The results obtained are shown in Fig. 19, where, following Jaensson et al. [74], the evolution of the distance between the outer and middle particle centers, $d$, and the angular velocity of the outer particles, $\omega_{z}$, are plotted as a function of strain. For all three meshes at short times (when the particle separation is large) the angular velocity is initially small and grows as the particles approach each other, before passing through a maximum at $t \dot{\gamma} \approx 1$. For the coarsest mesh, dyM_max $=0, \omega_{z}$ continues to grow after $t \dot{\gamma}=6$ until $t \dot{\gamma}=13$, at 


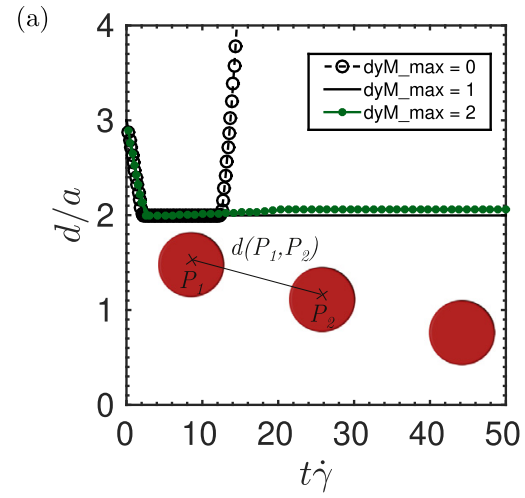

(b)

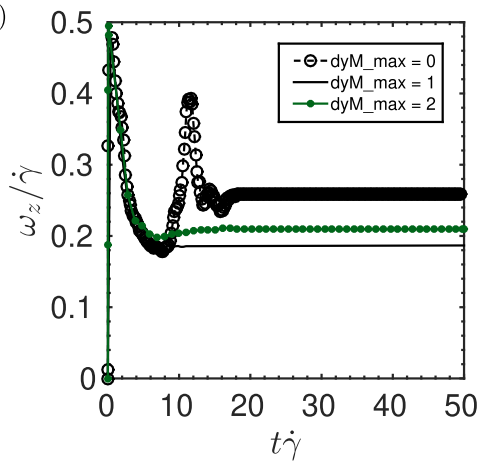

Fig. 19. Mesh refinement convergence study for the (a) distance, $d$, between outer and middle particle centers and the (b) angular velocity, $\omega_{z}$, of the outer particles. Here $2 a / H=1 / 4, R e=0.1, W i=3, \alpha=0.1, \beta=0.1$ and three particles are simulated.

Table 3

Total number of mesh cells and degrees of freedom (DOF) for the meshes employed to study particle alignment in viscoelastic fluids under steady planar shear flow.

\begin{tabular}{llll}
\hline $\begin{array}{l}\text { Domain } \\
L \times W \times H\end{array}$ & $\begin{array}{l}\text { Total number } \\
\text { of mesh cells }\end{array}$ & $\begin{array}{l}\text { Estimated number of cells } \\
\text { covered by the particles (solid fraction) }\end{array}$ & DOF \\
\hline $40 a \times 10 a \times 16 a$ & 51200 & 2144 & 524864 \\
$20 a \times 10 a \times 8 a$ & 12800 & 2144 & 140864 \\
\hline
\end{tabular}

which point the particles have minimum separation, and subsequently will pass each other, $d \gg 2 a$. However, for dyM_max $=\{1,2\}$, the angular velocity of the outer particles, after going through a single maximum at the beginning of the flow, reaches a steady value, at around $t \dot{\gamma}=16$. At this point, based on the results obtained with M2 and M3, the particles have formed a stable string with $d \approx 2 a$. Consequently, since the occurrence of particle alignment is the main subject of this case study, mesh M1 is clearly too coarse. Additionally, in order to evaluate the accuracy of M2 and M3, we obtained the extrapolated value of the steady-state particle distance and angular velocity, denoted by $d_{f}$ and $\left(\omega_{z}\right)_{f}$, respectively, to an infinitesimal grid size, using the Richardson's extrapolation technique [64]. Table 2 presents the calculated and extrapolated steadystate values and the relative errors obtained. As shown, the values of $d_{f}$ and $\left(\omega_{z}\right)_{f}$ appear to be appropriately accurate for M3 (dyM_max = 2 ), with relative errors of $1.0 \%$ and $3.7 \%$, respectively. Thus, the remaining simulations were performed with dyM_max $=2$, which offers a good trade-off between accuracy and efficiency (notice that the computational time needed for dyM_max $=2$ was, approximately, 6 days, and the estimated computational time for a refinement level of dyM_max $=$ 3 is about 45 days).

Subsequently, the effect of rheology and blockage ratio on particle alignment is studied. Table 3 gives the total number of cells and degrees of freedom (DOF), see Section 4.2 for details on how DOF is calculated, corresponding to each of the computational domains studied. (a)

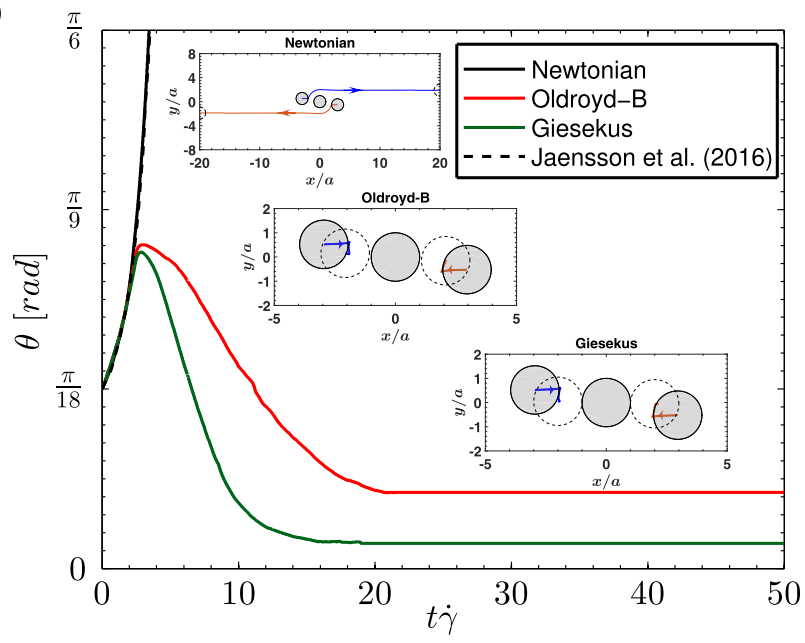

(b)

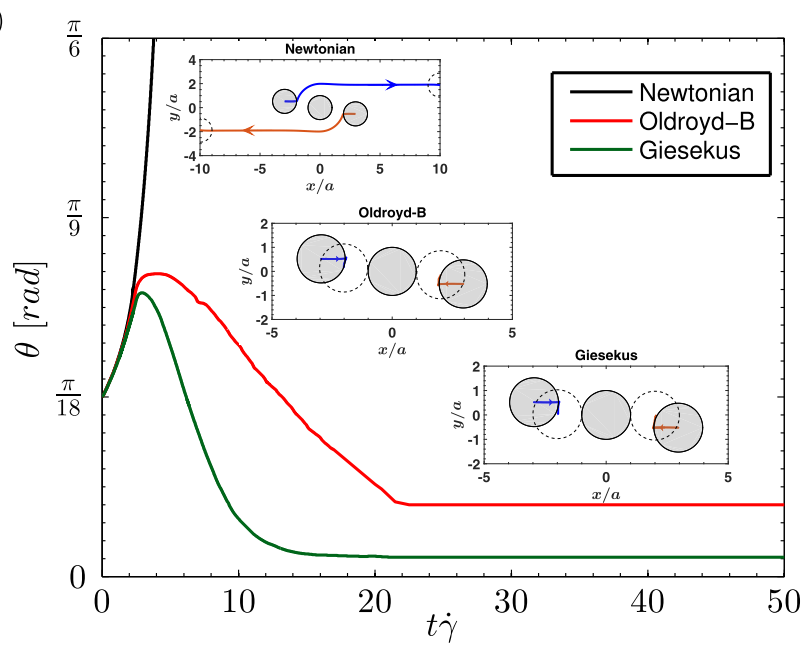

Fig. 20. Effect of the blockage ratio $2 a / H$ and fluid rheology on the angle $\theta$ evolution for the simulation of three particles suspended in Newtonian, Oldroyd-B and Giesekus fluids. The insets show the particle trajectories for each fluid model used. The shadowed gray and dashed drawings denote, respectively, the initial and final particle positions after 30 strain units of shear. (a) $2 a / H=1 / 8$ (b) $2 a / H=1 / 4$.

Additionally, a dynamic mesh refinement level at the particle interface is used to improve the accuracy of the numerical results. The Eulerian and Lagrangian time-steps used were $d t_{E u l}=10^{-6} \mathrm{~s}$ and $d t_{L a g}=10^{-7} \mathrm{~s}$, respectively. All computations were performed in parallel using 16 cores on a computer with a $2.60 \mathrm{GHz}$ E5-2650v2 Dual CPU processor and $64 \mathrm{~GB}$ of RAM. For the larger domain case study (51200 cells and 524864 DOF) the computational time taken by the newly-developed algorithm to reach $t \dot{\gamma}=30$ was approximately 12 days for the shear-thinning fluid model.

The results obtained in the numerical simulations are presented in Fig. 20, where the evolution of $\theta$ is shown as a function of strain. Following Jaensson et al. [74] if the angle $\theta$ remains constant or is still decreasing at $t \dot{\gamma}=30$, we will assume that the particles have aligned in a stable string. Concerning the effect of rheology, the results obtained are in accordance with the literature. There is no particle alignment in the absence of normal stress differences, with Newtonian fluids. For this case, the angle $\theta$ keeps increasing for all the shear strains and the particles move apart (see the insets for Newtonian fluids in Fig. 20). Good agreement with the results of Jaensson et al. [74] is also shown in Fig. 20. However, the presence of normal stress differences promote particle alignment [74], both for Oldroyd-B and Giesekus fluids. Shear-thinning further enhances the phenomena $[71,75]$, thus, 


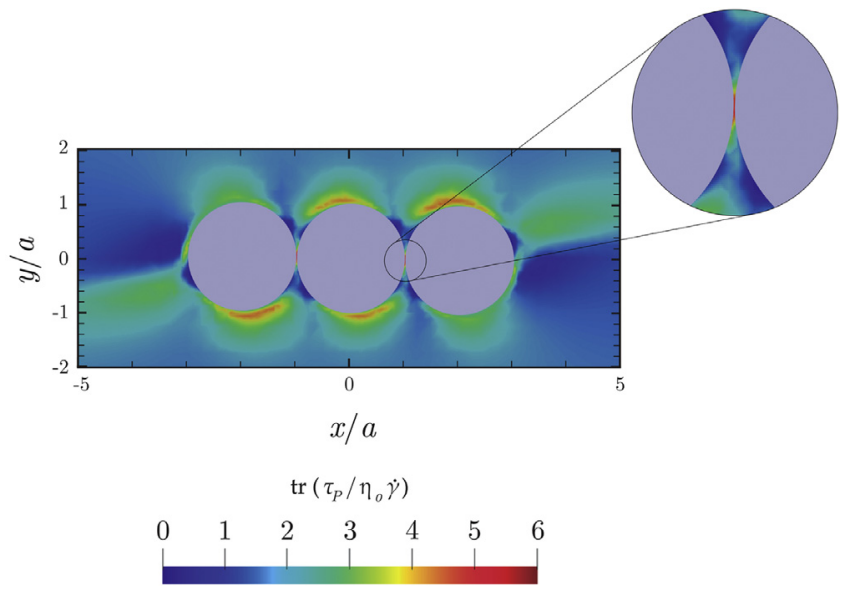

Fig. 21. Polymer stress contours for the alignment of three spherical particles suspended in a Giesekus fluid ( $W i=3, \alpha=0.1$ and $\beta=0.1$ ) under steady planar shear flow (blockage ratio $2 a / H=1 / 4$ ) at steady-state.

inducing a faster alignment with the Giesekus fluid when compared with the Oldroyd-B counterpart. For example, for the domain with blockage ratio $2 a / H=1 / 8$ the final angle obtained with the Giesekus fluid is $\theta_{f}=0.026 \mathrm{rad}$ compared to $\theta_{f}=0.074 \mathrm{rad}$ obtained with the Oldroyd-B fluid. Regarding the effect of the blockage ratio we can conclude that the rate of shear-induced particle alignment in the flow direction is enhanced by decreasing the channel gap, as shown by the reduction of both the maximum and steady-state orientation angles obtained with the viscoelastic fluids. For the Oldroyd-B and Giesekus fluids the steadystate angle decreases from $\theta_{f}=0.074 \mathrm{rad}$ to $\theta_{f}=0.070 \mathrm{rad}$ and from $\theta_{f}=0.026 \mathrm{rad}$ to $\theta_{f}=0.017 \mathrm{rad}$, respectively, corresponding to $5.4 \%$ and $34.6 \%$ reduction with the increase of the blockage ratio, respectively. Therefore, we can conclude that, when the gap decreases, the effect of shear-thinning in the particle alignment is stronger than the one promoted by the viscoelastic constant shear viscosity model. Another interesting point for discussion is that the computational stiffness of the calculations increases with the decrease of the gap size. In fact, the computational (wall-clock) times per iteration for the simulations with the Giesekus fluid using the two blockage ratios tested were, approximately, 0.15 and $0.45 \mathrm{~s}$ for $2 a / H=1 / 4$ and $2 a / H=1 / 8$, respectively. Therefore, considering a linear scale up of the computational time with the number of mesh cells (given in Table 3), we obtained $33 \%$ more computational time for $2 a / H=1 / 4$ than the expected $0.45 / 4=0.1125$ seconds per iteration (notice that 4 is the ratio between the number of mesh cells of the larger and smaller gap problems).

In Fig. 21 the trace of the polymer stress tensor is shown for the case of three spherical particles suspended in a Giesekus fluid ( $W i=$ $3, \alpha=0.1$ and $\beta=0.1$ ) under steady planar shear flow for the blockage ratio $2 a / H=1 / 4$ at steady-state $t \dot{\gamma}=30$ and a final orientation angle $\theta_{f}=0.017 \mathrm{rad}$. Regions of large polymer stresses are observed in the narrow fluid gaps (with minimum size of $h_{\min } / a=0.0625$ ) around the center particle, in agreement with Jaensson et al. [74]. The zoomed area in Fig. 21 shows the large stresses in the gap between the spherical particles, which contribute to the stability of the shear-aligned cluster of particles.

\section{Conclusions}

A numerical formulation for fully-resolved simulation of solid particles in viscoelastic fluids was presented and implemented using a combination of the finite-volume and the immersed boundary methods. The implementation was carried out by extending the open-source CFDEMcoupling framework, and the overall algorithm procedure solves the flow equations, including the viscoelastic constitutive equation using a log-conformation approach, with a PISO (Pressure-Implicit with Splitting of Operators) method, and an immersed boundary algorithm to model the flow around discrete force-free and torque-free particles present in the flow domain. This approach guarantees a tight coupling between the dynamics of the fluid and the solid discrete phase, by imposing a two-way coupling between the two phases. The coupling is provided by the calculation of the net hydrodynamic forces and torques exerted by the fluid on the solid particles. The algorithm subsequently updates the particle movement, comprising linear and rotational motions, which in turn provides new boundary conditions for the fluid domain.

The newly-developed algorithm was assessed in terms of accuracy in four benchmark studies. First, for the sedimentation of a sphere in a bounded rectangular box filled with a Newtonian fluid, the particle steady velocity was compared with experimental and numerical data available in the literature for different blockage ratios, and good agreement was obtained. Subsequently, the viscoelastic immersed boundary (IB) solver was tested on the sedimentation of a spherical particle through a FENE-CR fluid. The spatial and temporal orders of convergence for the algorithm were evaluated using different transient and advection discretization schemes, for which it was concluded that the theoretical orders of convergence of the tested schemes are retained by the new code. The velocity profiles of the sedimenting particle falling through a FENE-CR and Oldroyd-B fluids were also compared with literature results given by Rajagopalan et al. [42], and again an excellent agreement was obtained. Finally, the sedimentation of a spherical particle through a Giesekus fluid was computed for different retardation ratios and Weissenberg (Wi) numbers, in order to test the robustness of the numerical algorithm, specially for high Wi problems, which required the use of the log-conformation approach to achieve converged results. For single settling spheres, velocity overshoots and negative wakes were both observed at high Wi.

We also demonstrated the capability of the algorithm to predict the dynamics of a freely-suspended particle, by performing simulations of a sphere subjected to steady shear flow in a rectangular box filled with an Oldroyd-B fluid. The particle rotation rate, obtained by using two different approaches (fixed-mesh and immersed boundary), was compared with the numerical and experimental results presented by Snijkers et al. [43]. The results predicted by the developed code are in agreement with the ones available in the literature, where a steady decrease in the rotation rate was observed with increasing Weissenberg number.

The newly-developed viscoelastic immersed boundary code was also used to study the cross-stream migration of a neutrally buoyant sphere immersed in a pressure-driven Poiseuille flow, by using both Newtonian and viscoelastic constitutive models. The well known Segré-Silberberg effect [60], in which the spheres migrate to an equilibrium position between the tube centerline and the wall corresponding to $r_{s s} / R \approx 0.6$, was reproduced for the Newtonian fluid. For the viscoelastic fluid, the Giesekus constitutive model was used, and the effect of the retardation ratio on the particle equilibrium position was evaluated at $W i=1$. For particles released near the walls, increasing the retardation ratio leads the particles to move further outwards to a new equilibrium position of $r_{s s} / R \approx 0.65$, but for particles released near the tube center, increasing the retardation ratio has a non-monotonic effect on the particle equilibrium position. This non-monotonic trend of the results requires additional studies.

Finally, the capabilities of the developed code were tested in a challenging physical problem, the shear-induced particle alignment in wallbounded Newtonian and viscoelastic fluids. Simulations have been performed to illustrate the role of the fluid rheology (inelastic, elastic and shear-thinning) and blockage ratio on the alignment of three suspended particles. The results obtained show that the particle alignment occurs only in viscoelastic fluids, when normal stress differences are present, and the phenomena is enhanced by shear-thinning. Moreover, it was observed that the particle alignment rate increases as the gap is reduced and the blockage ratio increases. 
In summary, the results presented here show that the newly viscoelastic immersed boundary code, developed using an open-source framework, can accurately predict the flow patterns and particle motion in complex fluids for a number of benchmark complex flow problems, such as sedimentation, as well as particle rotation and translation in shear and Poiseuille flows. Furthermore, the combination of the new viscoelastic immersed boundary code with the log-conformation approach, and the use of unstructured and dynamic meshes in parallel, allows us to tackle new challenging problems, which have not been studied in the literature before, such as shear-induced particle alignment in wallbounded viscoelastic fluids.

\section{Acknowledgments}

This work is funded by FEDER funds through the COMPETE 2020 Programme and National Funds through FCT - Portuguese Foundation for Science and Technology under the project UID/CTM/50025/2013 as well as by the MIT Portugal Program (MPP). The authors would like to acknowledge the Minho University cluster under the project SearchON2: Revitalization of HPC infrastructure of UMinho (NORTE-07-0162FEDER-000086), co-funded by the North Portugal Regional Operational Programme (ON.2-0 Novo Norte), under the National Strategic Reference Framework (NSRF), through the European Regional Development Fund (ERDF). Additionally, the authors would like to acknowledge the Texas Advanced Computing Center (TACC) at The University of Texas at Austin for providing HPC resources that have contributed to the research results reported within this paper. http://www.tacc.utexas.edu Finally, the authors thank Bruno Santos from FSD blueCAPE Lda for insightful comments regarding the usage of the TACC resources.

\section{Supplementary material}

Supplementary material associated with this article can be found, in the online version, at 10.1016/j.jnnfm.2019.02.007.

\section{References}

[1] G.G. Stokes, Mathematical and Physical Papers, I-V, Cambridge University Press, 1901.

[2] J.F. Richardson, W.N. Zaki, The sedimentation of a suspension of uniform spheres under conditions of viscous flow, Chem. Eng. Sci. 8 (1954) 65-73, doi:10.1016/0009-2509(54)85015-9.

[3] G.K. Batchelor, Sedimentation in a dilute dispersion of spheres, J. Fluid Mech. 52 (1972) 245-268, doi:10.1017/S0022112072001399.

[4] A.J.C. Ladd, Effect of container walls on the velocity fluctuations of sedimenting spheres, Phys. Rev. Lett. 88 (2002), doi:10.1103/PhysRevLett.88.048301.

[5] C. Kloss, C. Goniva, A. Hager, S. Amberger, S. Pirker, Models, algorithms and validation for opensource DEM and CFD-DEM, Progress Comput. Fluid Dyn. 12 (2012) 140-152, doi:10.1504/PCFD.2012.047457.

[6] N.A. Patankar, P. Singh, D.D. Joseph, R. Glowinski, T.-W. Pan, A new formulation of the distributed lagrange multiplier/fictitious domain method for particulate flows, Int. J. Multiphase Flow 26 (2000) 1509-1524, doi:10.1016/s0301-9322(99)00100-7.

[7] A.A. Shirgaonkar, M.A. MacIver, N.A. Patankar, A new mathematical formulation and fast algorithm for fully resolved simulation of self-propulsion, J. Comput. Phys. 228 (2009) 2366-2390, doi:10.1016/j.jcp.2008.12.006.

[8] Z. Yu, N. Phan-Thien, Y. Fan, R.I. Tanner, Viscoelastic mobility problem of a system of particles, J. Non-Newtonian Fluid Mech. 104 (2002) 87-124, doi:10.1016/S0377-0257(02)00014-9.

[9] S. Chen, N. Phan-Thien, B.C. Khoo, X.J. Fan, Flow around spheres by dissipative particle dynamics, Phys. Fluids 18 (2006) 1-14, doi:10.1063/1.2360421.

[10] S. Padhy, E.S.G. Shaqfeh, G. Iaccarino, J.F. Morris, N. Tonmukayakul, Simulations of a sphere sedimenting in a viscoelastic fluid with cross shear flow, J. Non-Newtonian Fluid Mech. 197 (2013) 48-60, doi:10.1016/j.jnnfm.2013.02.003.

[11] S. Padhy, M. Rodriguez, E.S.G. Shaqfeh, G. Iaccarino, J.F. Morris, N. Tonmukayakul, The effect of shear thinning and walls on the sedimentation of a sphere in an elastic fluid under orthogonal shear, J. Non-Newtonian Fluid Mech. 201 (2013) 120-129, doi:10.1016/j.jnnfm.2013.07.007.

[12] S. Krishnan, E.S.G. Shaqfeh, G. Iaccarino, Fully resolved viscoelastic particulate simulations using unstructured grids, J. Comput. Phys. 338 (2017) 313-338, doi:10.1016/j.jcp.2017.02.068.

[13] C.S. Peskin, Flow patterns around heart valves: a numerical method, J. Comput. Phys. 10 (1972) 252-271, doi:10.1016/0021-9991(72)90065-4.

[14] C.S. Peskin, Numerical analysis of blood flow in the heart, J. Comput. Phys. 25 (1977) 220-252, doi:10.1016/0021-9991(77)90100-0.
[15] Z.Y. Zhou, S.B. Kuang, K.W. Chu, A.B. Yu, Discrete particle simulation of particlefluid flow: model formulations and their applicability, J. Fluid Mech. 661 (2010) 482-510, doi:10.1017/S002211201000306X.

[16] R. Glowinski, T.-W. Pan, T.I. Hesla, D.D. Joseph, J. Periaux, A fictious domain method with distributed Lagrange multipliers for the numerical simulation of particulate flow, Contemporary Mathematics 218 (1998).

[17] R. Glowinski, T.-W. Pan, T.I. Hesla, D.D. Joseph, J. Periaux, A distributed Lagrange multiplier/fictious domain method for the simulation of flow around moving rigid bodies: application to particulate flow, Comput. Methods Appl. Mech. Eng. 184 (2000) 241-267, doi:10.1016/S0045-7825(99)00230-3.

[18] R. Glowinski, T.-W. Pan, T.I. Hesla, D.D. Joseph, J. Periaux, A fictitious domain approach to the direct numerical simulation of incompressible viscous flow past moving rigid bodies: application to particulate flow, J. Comput. Phys. 169 (2001) 363-426, doi:10.1006/jcph.2000.6542.

[19] A. Hager, CFD-DEM on Multiple Scales - An Extensive Investigation of Particle-fluid Interactions, Christian Doppler Laboratory, Johannes Kepler Universitat Linz, 2014 Ph.D. thesis.

[20] C. Diaz-Goano, P.D. Minev, K. Nandakumar, A fictitious domain/finite element method for particulate flows, J. Comput. Phys. 192 (2003) 105-123, doi:10.1016/S0021-9991(03)00349-8.

[21] Z. Yu, X. Shao, A direct-forcing fictitious domain method for particulate flows, J. Comput. Phys. 227 (2007) 292-314, doi:10.1016/j.jcp.2007.07.027.

[22] J.J. Derksen, S. Sundaresan, Direct numerical simulations of dense suspensions: wave instabilities in liquid-fluidized beds, J. Fluid Mech. 587 (2007) 303-336, doi:10.1017/S0022112007007094

[23] A. ten Cate, C.H. Nieuwstad, J.J Derksen, H.E.A. Van den Akker, PIV experiments and lattice-Boltzmann simulations on a single sphere settling under gravity, Phys. Fluids 14 (2002) 4012-4025, doi:10.1063/1.1512918.

[24] C. Bodart, M.J. Crochet, The time-dependent flow of a viscoelastic fluid around a sphere, J. Non-Newtonian Fluid Mech. 54 (1994) 303-329, doi:10.1016/0377-0257(94)80029-4.

[25] H. Binous, R.J. Phillips, Dynamic simulation of one and two particles sedimenting in viscoelastic suspensions of FENE dumbbells, J. Non-Newtonian Fluid Mech. 83 (1999) 93-130, doi:10.1016/S0377-0257(98)00141-4.

[26] P. Singh, D.D. Joseph, T.I. Hesla, R. Glowinski, T.-W. Pan, A distributed Lagrange multiplier/fictitious domain method for viscoelastic particulate flows, J. NonNewtonian Fluid Mech. 91 (2000) 165-188, doi:10.1016/S0377-0257(99)00104-4.

[27] A.M. Ardekani, D.D. Joseph, D. Dunn-Rankin, R.H. Rangel, Particle-wall collision in a viscoelastic fluid, J. Fluid Mech. 633 (2009) 475-483, doi:10.1017/S0022112009990632.

[28] G. Li, G.H. McKinley, A.M. Ardekani, Dynamics of particle migration in channel flow of viscoelastic fluids, J. Fluid Mech. 785 (2015) 486-505, doi:10.1017/jfm.2015.619.

[29] G.-J. Li, A. Karimi, A.M. Ardekani, Effect of solid boundaries on swimming dynamics of microorganisms in a viscoelastic fluid, Rheol. Acta 53 (2014) 911-926, doi:10.1007/s00397-014-0796-9.

[30] G. Li, A.M. Ardekani, Undulatory swimming in non-newtonian fluids, J. Fluid Mech. 784 (2015) 1-13, doi:10.1017/jfm.2015.595.

[31] M.M. Villone, G. D'Avino, M.A. Hulsen, F. Greco, P.L. Maffettone, Simulations of viscoelasticity-induced focusing of particles in pressure-driven micro-slit flow, J. Non-Newtonian Fluid Mech. 166 (2011) 1396-1405, doi:10.1016/j.jnnfm.2011.09.003.

[32] N. Goyal, J.J. Derksen, Direct simulations of spherical particles sedimenting in viscoelastic fluids, J. Non-Newtonian Fluid Mech. 183 (2012) 1-13, doi:10.1016/j.jnnfm.2012.07.006.

[33] CFDEMcoupling, CFDEM project, 2011. https://www.cfdem.com/cfdemcoupling

[34] A. Hager, C. Kloss, S. Pirker, C. Goniva, Parallel resolved open source CFD-DEM: method, validation and application, J. Comput. Multiphase Flows 6 (2014) 13-27, doi:10.1260/1757-482X.6.1.13.

[35] K.I. Aycock, R.L. Campbell, K.B. Manning, B.A. Craven, A resolved two-way coupled CFD/6-DOF approach for predicting embolus transport and the embolustrapping efficiency of IVC filters, Biomech. Model Mechanobiol. 16 (2017) 851-869, doi:10.1007/s10237-016-0857-3.

[36] M.D. Chilcott, J.M. Rallison, Creeping flow of dilute polymer solutions past cylinders and spheres, J. Non-Newtonian Fluid Mech. 29 (1988) 381-432, doi:10.1016/0377-0257(88)85062-6.

[37] J.G. Oldroyd, On the formulation of rheological equations of state, Proc. R. Soc. A: Math.Phys. Eng. Sci. 200 (1950) 523-541, doi:10.1098/rspa.1950.0035.

[38] H. Giesekus, A simple constitutive equation for polymer fluids based on the concept of deformation-dependent tensorial mobility, J. Non-Newtonian Fluid Mech. 11 (1982) 69-109, doi:10.1016/0377-0257(82)85016-7.

[39] R. Fattal, R. Kupferman, Constitutive laws for the matrix-logarithm of the conformation tensor, J. Non-Newtonian Fluid Mech. 123 (2004) 281-285, doi:10.1016/j.jnnfm.2004.08.008.

[40] R. Fattal, R. Kupferman, Time-dependent simulation of viscoelastic flows at high Weissenberg number using the log-conformation representation, J. Non-Newtonian Fluid Mech. 126 (2005) 23-37, doi:10.1016/j.jnnfm.2004.12.003.

[41] A. Miyamura, S. Iwasaki, T. Ishii, Experimental wall correction factors of single solid spheres in triangular and square cylinders and parallel plates, Int. J. Multiphase Flow 7 (1981) 41-46, doi:10.1016/0301-9322(81)90013-6.

[42] D. Rajagopalan, M.T. Arigo, G.H. McKinley, The sedimentation of a sphere through an elastic fluid part 2. transient motion, J. Non-Newtonian Fluid Mech. 65 (1996) 17-46, doi:10.1016/0377-0257(96)01450-4.

[43] F. Snijkers, G. D’Avino, P.L. Maffettone, F. Greco, M.A. Hulsen, J. Vermant, Effect of viscoelasticity on the rotation of a sphere in shear flow, J. Non-Newtonian Fluid Mech. 166 (2011) 363-372, doi:10.1016/j.jnnfm.2011.01.004. 
[44] T.-W. Pan, R. Glowinski, Direct simulation of the motion of neutrally buoyant balls in a three-dimensional Poiseuille flow, Comptes Rendus Mécanique 333 (2005) 884 895, doi:10.1016/j.crme.2005.10.006.

[45] C. Fernandes, M.S.B. Araujo, L.L. Ferrás, J.M. Nóbrega, Improved Both Sides Diffusion (iBSD): a new and straightforward stabilization approach for viscoelastic fluid flows, J. Non-Newtonian Fluid Mech. 249 (2017) 63-78, doi:10.1016/j.jnnfm.2017.09.008.

[46] OpenFOAM, The open source CFD toolbox, 2004.https://www.openfoam.com/

[47] F. Habla, M.W. Tan, J. Haßlberger, O. Hinrichsen, Numerical simulation of the viscoelastic flow in a three-dimensional lid-driven cavity using the log-conformation reformulation in OpenFOAM, J. Non-Newtonian Fluid Mech. 212 (2014) 47-62, doi:10.1016/j.jnnfm.2014.08.005.

[48] F. Pimenta, M.A. Alves, Stabilization of an open-source finite volume solver for viscoelastic fluid flows, J. Non-Newtonian Fluid Mech. 239 (2017) 85-104, doi:10.1016/j.jnnfm.2016.12.002.

[49] rheoTool, Toolbox to simulate GNF and viscoelastic fluid flows in OpenFOAM, 2017.https://github.com/fppimenta/rheoTool

[50] P.A. Cundall, O.D.L. Strack, A discrete numerical model for granular assemblies, Géotechnique 29 (1979) 47-65, doi:10.1680/geot.1979.29.1.47.

[51] A. Di Renzo, F.P. Di Maio, Comparison of contact-force models for the simulation of collisions in DEM-based granular flow codes, Chem. Eng. Sci. 59 (2004) 525-541, doi:10.1016/j.ces.2003.09.037.

[52] DCS Computing GmbH, LIGGGHTS Public Documentation, 2015. https://www.cfdem.com/media/DEM/docu/gran_model hertz.html.

[53] J.H. Ferziger, M. Peric, Computational Methods for Fluid Dynamics, Springer, 2001.

[54] C. Hirsch, Numerical Computation of Internal and External Flows. Volume 1: Fundamentals of Computational Fluid Dynamics, Second, Elsevier, 2007.

[55] H. Jasak, Error Analysis and Estimation for the finite Volume Method with Application to Fluid Flows, Imperial College, University of London, 1996 Ph.D. thesis.

[56] H. Jasak, Dynamic mesh handling in OpenFOAM, 47th AIAA Aerospace Sciences Meeting, 2009. Orlando, Florida

[57] DCS Computing GmbH, LIGGGHTS Public Documentation, 2015. https://www.cfdem.com/media/DEM/docu/Manual.html.

[58] L. Verlet, Computer experiments on classical fluids I. thermodynamical properties of Lennard-Jones molecules, Phys. Rev. 159 (1967) 98-103, doi:10.1103/PhysRev.159.98.

[59] R.I. Issa, Solution of the implicitly discretized fluid flow equations by operatorsplitting, J. Comput. Phys. 62 (1986) 40-65, doi:10.1016/0021-9991(86)90099-9.

[60] G. Segré, A. Silberberg, Radial particle displacements in Poiseuille flow of suspensions, Nature 189 (1961) 209-210, doi:10.1038/189209a0.

[61] C.K. Aidun, Y. Lu, E.-J. Ding, Direct analysis of particulate suspensions with inertia using the discrete Boltzmann equation, J. Fluid Mech. 373 (1998) 287-311, doi:10.1017/S0022112098002493.
[62] M.J. King, N.D. Waters, The unsteady motion of a sphere in an elastico-viscous liquid, J. Phys. D. Appl. Phys. 5 (1972) 141-150.

[63] J. Happel, H. Brenner, Low Reynolds Number Hydrodynamics, Martinus Nijhoff, Dordrecht, 1983

[64] P.J. Roache, Quantification of uncertainty in computational fluid dynamics, Annu. Rev. Fluid Mech. 29 (1997) 123-160.

[65] M.A. Alves, P.J. Oliveira, F.T. Pinho, A convergent and universally bounded interpolation scheme for the treatment of advection, Int. J. Numer. Methods Fluids 41 (1972) 47-75, doi:10.1002/fld.428.

[66] O.G. Harlen, The negative wake behind a sphere sedimenting through a viscoelastic fluid, J. Non-Newtonian Fluid Mech. 108 (2002) 411-430, doi:10.1016/S0377-0257(02)00139-8.

[67] A. Einstein, Eine neue bestimmung der molejul dimensionen, Ann. Phys. 324 (1906) 289-306, doi:10.1002/andp.19063240204.

[68] G. D'Avino, M.A. Hulsen, F. Snijkers, J. Vermant, F. Greco, P.L. Maffettone, Rotation of a sphere in a viscoelastic liquid subjected to shear flow. Part I: simulation results, J. Rheol. 52 (2008) 1331-1346, doi:10.1122/1.2998219.

[69] S.A. Faroughi, C. Fernandes, J.M. Nóbrega, G.H. McKinley, A drag correction model for the inertia-less steady translation of a sphere in unconfined Oldroyd-B fluids, 2018. In preparation

[70] J. Michele, R. Pätzold, R. Donis, Alignment and aggregation effects in suspensions of spheres in non-Newtonian media, Rheol. Acta 16 (1977) 317-321, doi:10.1007/BF01523742.

[71] R. Scirocco, J. Vermant, J. Mewis, Effect of the viscoelasticity of the suspending fluid on structure formation in suspensions, J. Non-Newtonian Fluid Mech. 117 (2004) 183-192, doi:10.1016/j.jnnfm.2004.01.010.

[72] R. Pasquino, F. Snijkers, N. Grizzuti, J. Vermant, The effect of particle size and migration on the formation of flow-induced structures in viscoelastic suspensions, Rheol. Acta 49 (2010) 993-1001, doi:10.1007/s00397-010-0466-5.

[73] S. Van Loon, J. Fransaer, C. Clasen, J. Vermant, String formation in sheared suspensions in rheologically complex media: the essential role of shear thinning, J. Rheol. 58 (2014) 237-254, doi:10.1122/1.4853455.

[74] N.O. Jaensson, M.A. Hulsen, P.D. Anderson, Direct numerical simulation of particle alignment in viscoelastic fluids, J. Non-Newtonian Fluid Mech. 235 (2016) 125-142, doi:10.1016/j.jnnfm.2016.07.008.

[75] D. Won, C. Kim, Alignment and aggregation of spherical particles in viscoelastic fluid under shear flow, J. Non-Newtonian Fluid Mech. 117 (2004) 141-146, doi:10.1016/j.jnnfm.2004.01.005. 